\title{
Electronic Tickets, Smart Cards, and Online Prepayments: When and How to Advance Sell
}

\author{
Jinhong Xie • Steven M. Shugan \\ Marketing Department, Warrington College of Business Administration, 212 Bryan Hall, \\ University of Florida, Gainesville, Florida 32611 \\ xiej@notes.cba.ufl.edu \\ shugan@dale.cba.ufl.edu
}

\section{Abstract}

Advance selling occurs when sellers allow buyers to purchase at a time preceding consumption (Shugan and Xie 2000). Electronic tickets, smart cards, online prepayments, and other technological advances make advance selling possible for many, if not all, service providers. These technologies lower the cost of making complex transactions at a greater distance from the seller's site. They also give sellers more control over advance selling by decreasing arbitrage. As technology enhances the capability to advance sell, more academic attention is vital. This paper strives to exploit these technologies by developing advance-selling strategies.

Until recently, advance-selling research focused on the airline industry and specific characteristics of that industry. These characteristics included the price insensitivity of late arrivals (e.g., business travelers) compared with early arrivals (e.g., leisure travelers), demand uncertainty across flights on the same day, and capacity constraints. Recent findings by Shugan and Xie (2000) show that advance selling is a far more general marketing tool than previously thought. It does not require these industry-specific characteristics. It only requires the existence of buyer uncertainty about future valuations. Moreover, sellers without the ability to price discriminate can use advance selling to improve profits to the level of first-degree price discrimination. This finding is important because buyers are nearly always uncertain about their future valuations for most services (e.g., the utility of next year's vacation or a future college education).

In this paper, we take the next step from Shugan and Xie (2000). We show that advance-selling profits do not come from buyer surplus, but from more buyers being able to purchase. We determine when and how to advance sell in a variety of situations, including situations with limited capacity, second-period arrivals, refunds, buyer risk aversion, exogenous credibility, continuous preference distributions, and premium pricing. We determine when advance selling improves profits and, when it does, how to set advance prices. We ask and answer seven questions. First, when should sellers advance sell? Second, how much can advance selling improve profits compared with only spot selling? Third, what factors impact the profitability of advance selling and how? Fourth, should advance prices be higher or lower or the same as spot prices? Fifth, how do capacity constraints impact advance-selling strategies? Sixth, should sellers limit the number of advance sales? Finally, what is the possible impact of buyer risk aversion?

First, we provide precise conditions when sellers should advance sell. For example, without capacity constraints, we show that sellers should advance sell when marginal costs are sufficiently low to make it profitable to sell to buyers with low valuations and suffi- ciently high to convince buyers that the spot price will be higher than the advance price.

Second, we find that advance selling can almost double the profits from optimal spot selling to early arrivals. We also show that advance selling has no impact on consumer surplus in markets with homogenous consumers and no capacity constraints. Therefore, advance selling can increase social welfare because seller profits increase.

Third, we find that two very important factors impacting the profitability of advance selling are seller credibility and marginal costs. Buyers only advance buy when they expect an advantage from advance buying over spot buying. Without capacity constraints, sellers must credibly convince buyers that the advance price is at a discount to the spot price. We show that this condition is met under different circumstances. For example, large marginal costs can create credibility because buyers believe that these costs will lead to high spot prices.

Fourth, we find (although optimal advance prices can be at a discount to the spot price) that sometimes a premium is optimal. Premiums are optimal when capacity is large (but limited) and marginal costs are not too large. Buyers advance purchase at a premium to spot prices when capacity is limited and spot prices are low. (Note that this is not a risk premium, and risk aversion is not required.) No prior research has suggested this strategy because that research relies on the assumption that early arrivals are more price sensitive than later ones. Without that assumption, premium advance pricing is sometimes optimal.

Fifth, we find that binding capacity constraints can impact the profitability of advance selling in opposite ways. On one hand, capacity constraints create seller credibility. Buyers believe that spot prices will be high when they know spot capacity is limited (and, perhaps, more limited by advance sales). On the other hand, when capacity is limited, the need to increase sales from discounted advance prices diminishes.

Sixth, consistent with Desiraju and Shugan (1999) we find that limiting advance sales can be profitable, but only under restrictive conditions. These conditions are: (1) selling to all early arrivals would leave insufficient capacity in the spot period to sell to all secondperiod arrivals with high valuations, (2) the optimal spot price is high, and (3) marginal costs are sufficiently small to make advance selling profitable.

Finally, we find that buyer risk aversion can sometimes increase the profitability of advance selling.

Our findings provide precise guidelines for a large number of service providers that will have the technical capability to advance sell. For those service providers, advance selling provides a creative pricing strategy that can potentially provide substantial improvements in profits.

(Pricing; Advance Selling; Advance Pricing; Tickets; State-Dependent Utility; Services Marketing; Dynamic Pricing) 


\section{Introduction}

\subsection{New Technologies and Advance Selling}

Technological advances are having a greater impact on marketing and the ability to implement marketing strategies (Shugan 1994). These advancements often provide new capabilities or lower the costs of existing capabilities. In particular, new technologies now allow service providers to (1) construct more complex transactions, often at lower costs, (2) complete complex transactions at a greater distance from the service provider's site, and (3) control the extent to which arbitrage occurs. These capabilities are particularly important when service providers engage in the practice of advance selling.

Advance selling occurs when sellers allow buyers to purchase at a time preceding consumption (Shugan and Xie 2000). Service providers can advance sell their services in periods preceding the consumption period by using advance tickets, stamps, tokens, personal vouchers, passes, or other certifications good for service consumption during a future time interval (i.e., the spot period). Advance prices, paid in the advance period, are also called forward prices, prepaid vouchers, supersaver prices, advance ticket prices, early discounted fares, early-bird specials, early booking fares, and advance purchase commitments.

Although some sellers have practiced advance selling, technological limitations have restricted its usefulness. For example, traditional tickets have allowed sellers to advance sell their services well before consumption, but the transaction costs were high. It was difficult and costly to make advance transactions from distant locations except with specialized channels (e.g., travel agencies). Traditional tickets were also limited to only the simplest and most standardized transactions. Traditional tickets suffered from many other disadvantages, including the overwhelming problem of arbitrage. The ability of arbitrageurs to resell advance tickets often dramatically decreased seller profitability.

New technologies are overcoming these problems with traditional tickets. They decrease transaction costs in making advance sales and allow far more complex transactions. They prevent or discourage ar- bitrage. They allow many new applications of advance selling. These new technologies include electronic tickets, smart cards, online prepayments, and electronic money. These technologies are making advance selling possible for many, if not all, service providers. Electronic tickets can store information on a magnetic strip, while smart cards actually hold the customer's information in a computer chip. They can be sold online and allow sellers tremendous flexibility. Restaurants, for example, are now able to advance sell an electronic ticket good for two meals, only on Tuesday nights, for up to $\$ 20$, at any participating chain for the next month, good only for a customer whose picture is electronically coded on the ticket. The electronic ticket is updated each time it is used, and all the restrictions are encrypted on the ticket. Sellers can also use smart cards, among many other advance-selling applications, such as electronic money. Some golf clubs, for example, now allow members to prepurchase services by loading smart cards with "money" that can be spent in the club shop or club bar.

With the development of Internet and smart card technologies, more service providers are beginning to use advance selling (e.g., see www.raileurope.com). Some travel agents sell prepaid vouchers for taxis, hotels, meals, and other travel services. Sheraton and Holiday Inn have prepaid voucher programs (Lollar 1992). Airlines sell discounted advance tickets. Conferences, lectures, tours, and seminars offer lower early registration fees. Some railroads and subways are beginning to offer discounted advance ticket prices. Outdoor concerts, rodeos, parades, festivals, playhouses, county fairs, civic events, circuses, and other events are experimenting with cheaper advance tickets (Melendez 1997, McVea 1997). New online services (see www.ticketmaster.com) offer advance sales from credit unions, AAA Travel, and Fox Box Office. They now allow remote buyers with palmtops to advance purchase tickets for fishing excursions, theater events, sports events, limousine services, music concerts, trains, golf vacations, cruises, amusement parks, theme parks, ${ }^{1}$ and Broadway plays.

${ }^{1}$ For example, Universal Studios and several Six Flags parks sells one-day passes, called "Twickets," that expire after 1 week. 
As technology continues to lower the cost of advance selling, more service providers will be able to administer sophisticated advance-selling strategies previously enjoyed only by large airlines and hotel chains. Rapid advances in information technology ${ }^{2}$ will allow more sellers to design advance-selling systems because they can better estimate advance demand with accurate buyer data and analysis. Moreover, more firms will be able to sell in advance by means of technologies like online purchase, smart tickets, and personalized vouchers. Whereas previously buyers usually needed to travel to the service provider to make a prepurchase, some providers now offer advance tickets and other prepayment through online websites (e.g., http://www.advancetickets.com and http://www. concoursedelegance.org/advanced_tickets.htm). Computerized ticket machines can record demand and change prices with very little cost. Smartcards-small reusable tickets containing computer chips that allow complex prepayments and fare collection (Strandberg 1992) - both reduce transaction costs (e.g., http:/ / www.smartaxis.co.uk) and limit arbitrage because personalized information stored in electronic form makes it relatively difficult to resell unused tickets. Improving technology may make advance selling possible in many new categories, including dry cleaning, dining, videos (Eliashberg et al. 1998), and products with network externalities (Padmanabhan et al. 1997, Xie and Sirbu 1995).

As technology enhances the capability to advance sell, more academic attention is vital. This paper strives to exploit these technologies by developing advance-selling strategies.

\subsection{The Goal and Findings of This Study}

Our paper's goal is to determine when advance selling improves profits and, when it does, how to set advance prices. We specify conditions under which advance selling is more profitable than spot-only strategies (i.e., tickets sold at the gate). We examine the possibility of selling in both an advance and spot period. We allow buyers to arrive in each period. We also allow early arrivals to advance buy or wait.

${ }^{2}$ For example, see: Technology for CED: Meaningful technology drives CED. Chain Store Age 72(1) S42, 1996.
We ask and answer seven questions. First, when should sellers advance sell? Second, how much can advance selling improve profits, compared with only spot selling? Third, what factors impact the profitability of advance selling, and how? Fourth, should advance prices be higher, lower, or the same as spot prices? Fifth, how do capacity constraints impact advance-selling strategies? Sixth, should sellers limit the number of advance sales? Finally, what is the possible impact of buyer risk aversion? We now summarize our answers to these questions and present some of our findings on optimal seller strategies.

First, given the technology, we conclude that advance selling is appropriate in more situations than previously identified. Similar to Shugan and Xie (2000), we find the profitability of advance selling is fundamental to buyer uncertainty about future valuations. This finding is important because buyers are nearly always uncertain about their future valuation of most services (e.g., the utility of next year's vacation or a future college education). Given buyer uncertainty and sufficiently low marginal costs, sellers can potentially earn greater profits by advance selling.

However, we also find that for advance selling to improve profits, buyers must expect an advantage from advance purchasing. That advantage could be either a discount over the spot price or capacity that would not be available in the spot period. Hence, although capacity constraints are not required, they can make advanced selling a viable strategy when credible discounting is not possible. Specific buyer risk preferences, limits on advance sales, and refunds can also act in a way to enhance the advantage of advance selling.

These findings suggest that advance selling can help service providers in any industry in which buyers have uncertainty about their future valuations for a service (provided announced spot prices are credible and marginal costs are within a specified range). As discussed later in detail, advance selling increases profits by increasing sales to more buyers (compensating for the discounted advance price) or increases profits by allowing a premium advance price.

Second, without any industry-specific factors, we 


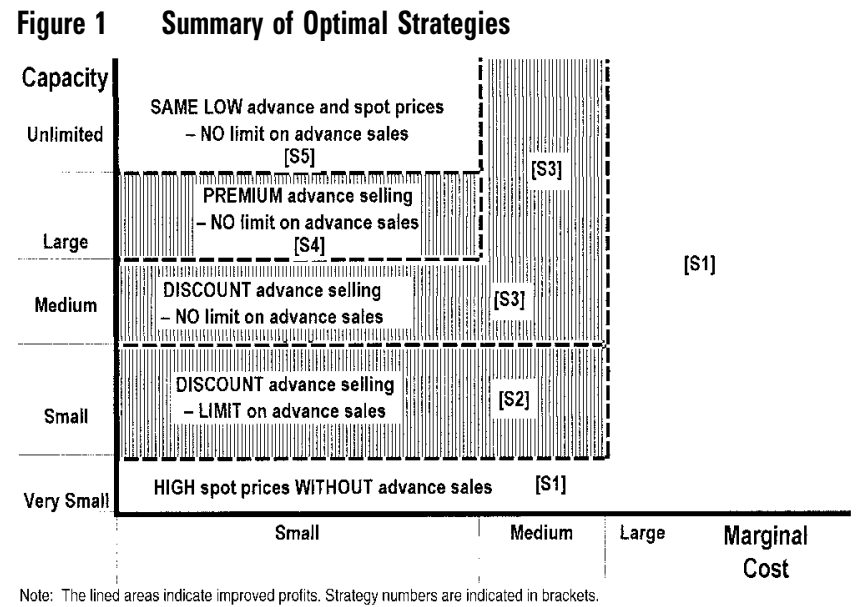

derive the profit improvement from advance selling to early arrivals. We find that the profits from advance selling to early arrivals can be up to twice the profits from only spot selling. Additional industryspecific factors (e.g., varying price sensitivities) could further increase the advance-selling profits, but they are not required.

Third, we find seller credibility and marginal costs are important factors that determine the profitability of advance selling. Buyers are willing to advance purchase when they believe future spot prices will be high (for reasons we discuss later). This situation occurs when seller announcements about future spot prices are credible. In addition to credibility, the advance price must improve seller profits over only spot selling. When advance prices are at a discount to the spot price, advance selling provides an increase in sales volume by selling to some buyers who would not purchase in the spot period because they realize low valuations. A low marginal cost ensures that the volume from additional purchases offsets the discount in the advance period.

Figure 1 shows the answers for questions 4 through 6. It summarizes our subsequent findings regarding the level of advance prices and how capacity constraints impact advance prices.

As Figure 1 shows, the optimal strategy depends on the level of capacity and marginal costs (these levels are precisely defined later). Five different selling strategies are sometimes optimal. Strategies [S2], [S3], and [S4], shown as shaded boxes, provide higher profits than only spot selling. Now, consider each of the five strategies.

[S1] HIGH Spot Prices WITHOUT Advance Sales. This strategy is to sell at a high spot price (inducing only some buyers to purchase) and to not advance sell. This strategy is best either when capacity is very small or when marginal costs are large. However, the reason is different for each case. When capacity is very small, the seller can sell all of the capacity at a high price. There is no incentive to offer advance discounts to increase sales because sales are limited by capacity. With large marginal costs, it is unprofitable to advance sell at discounted prices because the revenue from some buyers (i.e., those with future low valuations) does not exceed the marginal cost.

[S2] DISCOUNT Advance Selling-LIMIT on Advance Sales. This strategy involves selling in advance at a discount to the spot price, but limiting the number of advance sales to reserve sufficient capacity for sale in the spot period. The strategy is best when capacity is sufficiently small to make high spot prices optimal, and when marginal costs are sufficiently small so that advance selling at a discount is profitable. Consistent with the findings of Desiraju and Shugan (1999), we find that advance discounts with limited sales are only appropriate in specific situations. Only firms facing small capacity and less than large marginal costs should offer discounted advance prices and limit sales at those prices. With large capacity, limiting early sales provides no benefit because there is sufficient capacity to serve late arrivals. When capacity is very small, advance selling has no advantage because all sales should occur at high spot prices.

[S3] DISCOUNT Advance Selling-NO Limit on Advance Sales. This strategy is similar to Strategy [S2], except that advance sales are not limited. The reason for not reserving capacity is that when all early arrivals advance buy, capacity in the spot period is now sufficient to serve all customers with high valuations. This strategy is optimal in two cases, both cases having sufficiently large capacity (i.e., medium, large, or unlimited). 
The first case is when costs are medium. Here, costs are sufficiently high to make high spot prices credible but not too high to make discounting unprofitable. In that range, each advance sale increases profits. With medium capacity or more, sellers have sufficient capacity to serve all late arrivals with high valuations. The additional advance sales compensate for the advance discount. Hence, Strategy [S3] is optimal with either limited or unlimited capacity, provided that marginal costs are medium.

The second case is when costs are small. Here, Strategy [S3] is optimal only with a medium capacity because that capacity is required to create credibility.

Unlike prior research (Gale and Holms 1993, Dana 1999, Desiraju and Shugan 1999) that motivated advance selling as a consequence of capacity constraints, we find advance selling can increase profits with unlimited capacity. Advance selling improves profits by inducing sales from all early arrivals, including those who would not have spot purchased. With this strategy, no limit on advance sales is necessary because capacity is sufficient to allow all late arrivals to purchase at the high spot price. Note that this strategy requires credibility, i.e., Period 1 arrivals only advance buy when they believe spot prices will be high. Unlimited capacity requires sufficiently large marginal costs to ensure a high spot price. Limited capacity, in contrast, creates its own credibility and therefore allows advance selling for a wider range of costs.

[S4] PREMIUM Advance Selling-NO Limit on Advance Sales. This strategy is to advance sell at a premium to the spot price. This strategy is best when capacity is large (i.e., sufficient to make a low spot price optimal but insufficient to satisfy all spot demand). Here, some buyers pay a premium in the advance period over the spot price. They pay this premium because advance buying has a higher expected buyer surplus than waiting. The expected surplus from waiting is smaller because not finding spot capacity produces no surplus. Note that this result is not a risk premium, and risk aversion is not required. Buying at a premium simply has a higher expected value than waiting. By charging a premium, sellers improve profits compared with spot selling only.
Most previous research (Gale and Holmes 1993, Dana 1999, Desiraju and Shugan 1999) has only considered advance selling at a discount because advance selling was motivated by a different reason, i.e., early arrivals are more price sensitive. Our results reveal that advance selling at a premium price can improve profits over only spot selling without assuming specific patterns of price sensitivity.

[S5] SAME LOW Advance and Spot Prices. This strategy is to advance and spot sell at a low price that induces purchases from all buyers. This strategy is best when there is unlimited capacity and low costs. Without capacity constraints or high marginal costs, only a low spot price is credible. Hence, buyers will only advance buy at prices equal to the low spot price. Advance selling at that price generates no more profit than only spot selling.

Finally, our last question concerns the possible outcome of buyer risk aversion. We find that buyer risk aversion can sometimes increase the profitability of advance selling. To understand this, consider the possible risks. Buyers who advance purchase and later have low valuations lose by paying more than their valuations. Buyers who do not advance purchase lose the discount from advance buying when they later have high valuations. When risk aversion accentuates the latter loss over the former loss, the profitability of advance selling increases.

Our findings provide clear guidelines for managers who are acquiring the technical capability to advance sell or are in industries where new technologies are emerging. In these cases, advance selling provides a creative pricing strategy that can potentially increase profits.

We proceed as follows. We first review the extant literature and develop a model for analyzing advance selling. We examine the impact of capacity constraints, risk-averse buyers, and refunds on advance selling. The paper ends with conclusions and areas for future research.

\section{Existing Literature}

There are several related literatures. First we consider the literature on price discrimination in the airline 
industry, which focuses on specific features of that industry, including limited capacity and late arrivals who are less price sensitive than earlier arrivals (Gale and Holmes 1993, Dana 1999). The late arrivals are sometimes modeled as having a higher cost of commitment. Desiraju and Shugan (1999) argue that these factors suggest that airlines should limit advance sales to reserve capacity for the consumption period, and that yield management systems are sophisticated systems for limiting, rather than encouraging, advance sales. Yield management systems (YMS)—also called capacity management-implement sophisticated heuristics and tools for capacity allocation. These tools include overbooking capacity, reserving capacity, and estimating available capacity. Overbooking with opportunistic cancellations can significantly improve firm profits (Biyalogorski et al. 1999).

Our paper significantly generalizes the airline-pricing literature. As noted earlier, advance selling improves profits when sellers can credibly convince buyers that the advance price is at a discount to the spot price. The high spot price encourages price-sensitive buyers to advance purchase, resulting in price discrimination. The specific features of the airline industry, including capacity constraints and late priceinsensitive arrivals, allow price discrimination by making high spot prices credible. However, we show that the profit improvements from advance selling do not depend on capacity constraints or a negative correlation between arrival time and price sensitivity. Price discrimination is not required. Hence, although yield management systems may be limited to capacity-constrained services (Desiraju and Shugan 1999), we show that industries not sharing the industry-specific factors of airlines can still improve profits with advance selling.

A second related body of literature is that on price deals (or sales promotions). In their seminal article, Blattberg, Eppen and Lieberman (1981) suggest that buyers, with low inventory costs, stock items on deal for future consumption, thereby transferring seller inventory-carrying costs to buyers. Later research (Jeuland and Narasimhan 1985) suggest that this transfer creates price discrimination because buyers with higher carrying costs pay higher prices. This reason for promotions is consistent with later findings showing that market shares remain unaltered by promotions (Lal and Padmanabhan 1995). Our paper does not require differences in inventory-carrying cost or price discrimination between heterogeneous buyers.

A third related literature considers contingent claims contracts and the special case of insurance. The execution of these contracts depends on risky but observable events (Malinvaud 1972, p. 10). For example, futures markets let buyers and sellers hedge against observable future price changes (Working 1953), using contingent futures contracts. Another example is fire insurance for which insurers pay benefits only when fires occur. Lotteries are also contingent contracts that pay prizes only when buyers' numbers are chosen. Here, sellers' costs depend on whether the event occurs. Unlike our paper, in which sellers do not observe buyers' consumption states, contingent claims require sellers to observe the contracted event (i.e., the fire). Also, with contingent claims, services are only rendered when both buyers and sellers observe the event. Finally, unlike in this study, where information is asymmetric in the consumption period, contingent claims contracts assume symmetry as everyone learns spot prices together.

Note that airline reservations may be contingent claims contracts for risk-averse buyers. For example, with an interesting two-period model, Png (1989) argues that airline reservations are a form of insurance. He exploits the fact that airlines are a faster, but uncertain, mode of travel. Future travel by air involves uncertainty about fares and overbooking. The alternative, i.e., the train, is a certain but slower means of travel that is not possible in the future. Risk-averse buyers are reluctant to forgo current and certain travel in favor of risky future but faster travel. Free airline reservations that guarantee the future price provide the required insurance that encourages buyers to wait and fly. Our paper shows that advance selling can improve profits without insurance, risk-averse buyers, overbooking, unavailable future alternatives, or other airline-specific factors.

A very interesting paper by Hayes (1987) uses aspects from the literatures on insurance and price dis- 
crimination. Hayes shows that two-part tariffs (which act as price-discrimination schemes) can enhance risk-averse-buyer welfare by acting as insurance. Buyers pay a fixed amount as insurance and then consume at below marginal costs after consumption uncertainty is resolved. The benefit of insurance allows Hayes (1987) to explain how two-part tariffs can persist in highly competitive markets that should eliminate price discrimination.

A fourth related and extensive literature concerns peak-load pricing. This literature focuses on how prices should change when there are seasonal shifts in demand. For example, Radas and Shugan (1998b) show that encouraging consumers to shift their demand to off-peak periods is unprofitable but sometimes socially desirable. This literature examines the implications of fluctuating demand rather than advance selling.

The most related literature is on state-dependent utility theory, where buyer valuations depend on future circumstances or state-dependent factors (e.g., Fishburn 1974, Kreps 1979, Zettelmeyer 1996). For example, a spare tire's value is greater in the state in which the driver gets a flat than when no flat occurs. A formal French dinner provides more value when a diner is in the mood for a formal French dinner than when the diner is not. Unlike traditional utility theory, where utility is only a function of product attributes (Hauser and Wernerfelt 1990, Johnson et al. 1995, Rust and Oliver 1994, Srinivasan et al. 1997), now utility also becomes a function of future circumstances. Common applications (e.g., Cook and Graham 1977) of state-dependent utility functions and extensions (e.g., Plummer and Harman 1986) occur in public policy and risk assessment.

The empirical marketing literature is consistent with state-dependent utilities. Simonson and Winer (1992) suggest that "it may be difficult to predict a week in advance whether one will be in the mood for a strawberry- or a raspberry-flavored yogurt." McAlister and Pessemier (1982) imply that predicting preferences is difficult because of variations in mood, usage situations, and so on. Bettman et al. (1998) note that situational factors cause preference changes and "although a consumer has a strong preference for chocolate, he or she may sometimes order another dessert." Situational factors as minor as ambient odor (Mitchell et al. 1995) or social surroundings (Stayman and Deshpande 1989) could change preferences. Econometric techniques allow the estimation of "heterogeneity caused by consumers changing their latent preference" (Bockenholt and Dillon 1997).

Shugan and Xie (2000) argue that state-dependent utility theory can be applied to situations in which consumption and purchase occur at different times. They argue that when purchase precedes consumption, buyers can be uncertain about their future utility from consumption. Consider buying a concert ticket two weeks before a concert. In advance, buyers may be uncertain about their future consumption state (e.g., health, expected conflicts, mood). At the time of the concert, buyer uncertainty about the consumption state is resolved, while sellers remain unable to observe buyer states. Consequently, information is symmetric in the advance period but asymmetric in the consumption period.

In the spot period, there is an information asymmetry because sellers are unable to observe buyer valuations. Valuations are private information known only by buyers. Sellers are, therefore, at a relative informational disadvantage (e.g., Gerstner and Hess 1987, Wernerfelt 1995). However, Shugan and Xie (2000) observe that the relationship is dynamic and changes over time. Moving forward from the consumption period, the asymmetry in information diminishes as buyers also become uncertain about their future consumption state. In the advance period, that seller disadvantage disappears because, like sellers, buyers also are uncertain about their future valuations. Shugan and Xie (2000) suggest that advance selling overcomes the informational disadvantage of sellers.

In the Shugan-Xie model buyers have probabilities $q$ and $1-q$ of having a high and low future valuations $H$ and $L$. They show that advance selling at price $q H+(1-q) L$ provides more profits than only spot selling at any spot price. They present the following example. Consider 100 potential ticket buyers for a county fair with low and high valuations $\$ 2$ and $\$ 5$, respectively. Suppose the valuations are equally likely 
and marginal costs are zero. When the fair only spot sells, the fair can sell tickets at $\$ 2$ to all buyers, or at $\$ 5$ to half the buyers. The $\$ 2$ and $\$ 5$ prices yield profits of $\$ 2 \times 100=\$ 200$ and $\$ 5 \times 1 / 2 \times 100=\$ 250$, respectively, so the optimal spot price is $\$ 5$. With advance selling weeks before the fair date, buyers have an expected valuation of $(\$ 2 \times 1 / 2)+(\$ 5 \times 1 / 2)=\$ 3.50$. Advance selling at $\$ 3.50$ produces profits of $\$ 3.50 \times$ $100=\$ 350$, which is greater than the spot profits at any spot price. The fair improves profits by $(\$ 350-$ $\$ 250) / \$ 250=40 \%$ without capacity constraints and without price discrimination (i.e., all buyers pay the same price).

The subsequent sections of this paper generalize the Shugan-Xie model to allow second-period arrivals, capacity constraints, premium pricing, limits on advance sales, buyer risk preferences, exogenous seller credibility, refunds, and other factors. Unlike prior research on advance pricing, we identify situations when the advance price should be higher than, lower than, or the same as the spot price.

We do not, however, consider the impact of competition on advance selling in this paper. In another paper (Shugan and Xie 2001) we do examine the impact of competition. We find that the market power of the seller determines the outcome of advance selling. However, when multiple sellers all advance sell, everyone can potentially gain, creating a "win-winwin" situation where each seller's profit and buyer surplus all increase.

\section{The Model}

Consider a two-period model where $N>0$ buyers arrive in each period. We refer to Periods 1 and 2 as the advance and spot periods, respectively. Consumption occurs only in the spot period, but purchase can occur in either period. Buyers and sellers are both forward looking and anticipate Period 2. In Period 1, buyers are uncertain about their consumption states and, hence, their valuation for the service in the spot period. So, buyers form expectations in Period 1 about their future valuations consistent with expected utility theory (Fishburn 1974).

Consider, for example, preregistration for a confer-
Figure 2 The Process

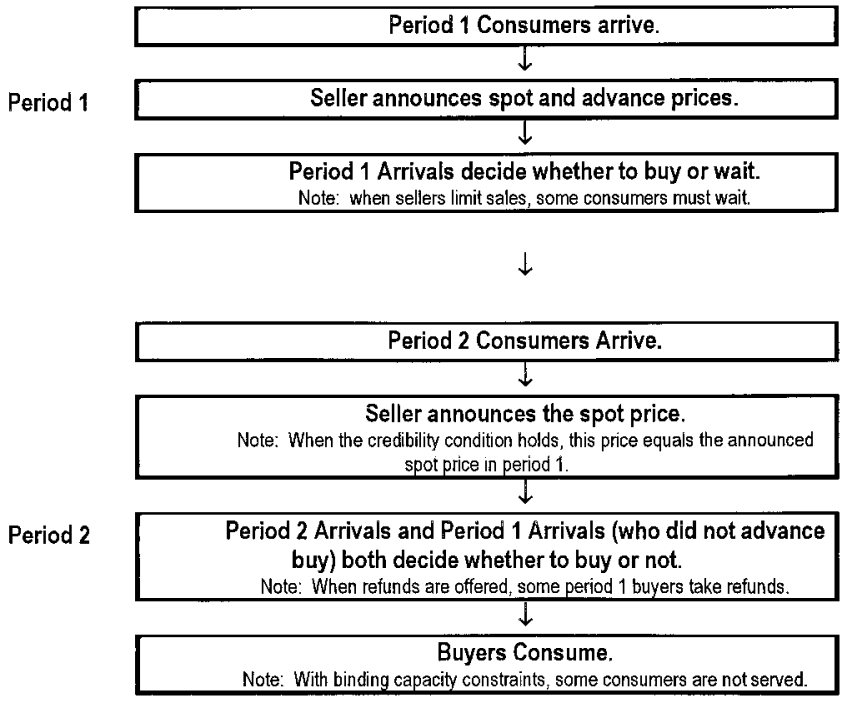

ence. At the time of the conference, possible attendees learn of conflicts, opportunities, and constraints that were uncertain at the time of preregistration. In favorable states, they will pay more than in unfavorable states. In Period 1, early arrivals learn of the conference and decide whether to preregister. They form expectations about how much they will value the conference. They compare the spot and advance price to decide whether to advance purchase. Those who do not advance buy can spot buy in Period 2, or not buy. Additional late arrivals in the spot period also learn of the conference and decide whether to register.

Note that uncertainty in buyer expectations comes from uncertainty about consumption states rather than product attributes. The former uncertainty is resolved by time alone, while the latter uncertainty is resolved after consumption. Although both types of uncertainty may be present, we emphasize the former.

\subsection{The Process}

Figure 2 depicts the events in the two-period process. Period 1 has three events: (1) Period 1 consumers arrive, (2) the seller announces the spot and advance prices, and (3) Period 1 arrivals decide whether to buy or wait. In the basic model, all arrivals can buy if they choose to do so. Later in this paper, we con- 
sider situations in which sellers limit sales because of limited capacity. Period 2 has four events: (1) Period 2 consumers arrive, (2) the seller announces the spot price (as we discuss later, rational buyers in Period 1 should anticipate this actual price when buyers make Period 1 buying decisions), (3) new arrivals, as well as Period 1 arrivals who did not advance buy, now decide whether or not to spot buy (as we discuss later, some advance buyers may receive refunds), and (4) buyers consume. In the basic model, all buyers consume because capacity is sufficient. Later, however, we allow limited capacity to prevent some buyers from consuming.

The problem is a dynamic program, solved by backward induction. We first determine buyer behavior in Period 2. That behavior implies optimal spot prices. Those prices determine buyer behavior in Period 1. Buyers in the advance period consider the advance price and the anticipated spot prices to decide whether to advance purchase or wait. That advance behavior dictates the optimal advance price. We begin with buyer behavior in the spot period.

\subsection{Buyer Behavior in the Spot Period}

In the spot period, consumption occurs and the buyer's utility depends on the consumption state (i.e., numerous uncertain factors such as the buyer's mood and possible conflicts occurring at the time of consumption). We do not restrict the duration of either the advance or the spot period. The later period could be a particular show time or several days in duration. Either period's duration is irrelevant for our qualitative results.

Following the Shugan-Xie basic model (Shugan and Xie 2000), we consider potential buyers facing two possible consumption states-favorable or unfavorable (i.e., a two-point Bernoulli distribution). The market consists of buyers who may purchase in either state, but will pay less in unfavorable states. Buyer valuations (i.e., the maximum amount buyers will pay) for a service in favorable and unfavorable states are $H$ and $L$, respectively. To be precise, these valuations are the differences in utility between consuming and not consuming in favorable and unfavorable states, respectively. By definition, valuations are greater in favorable states, i.e., $H>L$. Finally, $q$ denotes the probability that a buyer is in a favorable state.

Following convention, consumers buy when and only when they get a nonnegative surplus. Moreover, given several alternatives, they choose the one with the largest surplus. When the surplus is equal, buyers choose the alternative favoring the seller because sellers can resolve buyer indifference with an infinitesimal price reduction.

\subsection{Seller Strategy in the Spot Period}

Let $p_{t}$ denote price in period $t, t=1,2$. Demand in Period 2 comes from Period 2 arrivals as well as Period 1 arrivals who have not purchased in Period 1. Spot pricing above $H$ yields no demand. By spot pricing at $L$ or less, the seller can induce all consumers to buy. Other spot prices induce sales only from consumers in favorable states.

Using backward induction, we first find optimal spot prices, $p_{2}^{*}$, that maximize Period 2 profit, then solve the Period 1 problem. Let $c$ denote the seller's constant marginal cost. Theorem 1 follows. See the appendix for proofs.

Theorem 1 (Basic Model-Optimal Seller Strategy IN PerIod 2). The best strategy is to spot price at $p_{2}^{*}=H$ when $c \geq c$, and price at $p_{2}^{*}=L$, otherwise, where $c=(L$ $-q H) /(1-q)$.

\subsection{Buyer Behavior in the Advance Period}

In Period 1, buyers observe the advance price $p_{1}$ and expect a future spot price, denoted $\tilde{p}_{2}$. Buyers advance purchase only when the expected surplus from advance buying $(E S A)$ exceeds the expected surplus from waiting $(E S W)$. The expected valuation for the product or service is $E V=q H+(1-q) L$ and the expected surplus from advance buying is $E S A=E V$ $-p_{1}$. The expected surplus of waiting depends on whether $\tilde{p}_{2}>L$ or $\tilde{p}_{2} \leq L$. When $\tilde{p}_{2} \leq L$, then ESW $=$ $E V-\tilde{p}_{2}$. When $\tilde{p}_{2}>L$, then $E S W=q\left(H-\tilde{p}_{2}\right)$.

Buyers advance purchase when both (a) $E S A \geq$ $E S W$ and (b) $E S A \geq 0$. Theorem 2 follows.

Theorem 2 (Basic Model-Buyer Behavior in Period 1). When buyer valuations are $H$ and $L$ with probabilities 
$q$ and $1-q$, respectively, the maximum price inducing an advance purchase is

$$
p_{1}^{\max }= \begin{cases}q H+(1-q) L=E V & \text { if } \tilde{p}_{2} \geq H \\ q \tilde{p}_{2}+(1-q) L & \text { if } L \leq \tilde{p}_{2} \leq H \\ \tilde{p}_{2} & \text { if } \tilde{p}_{2} \leq L .\end{cases}
$$

Theorem 2 implies that buyers will advance buy when the price is no more than $p_{1}^{\max }$, which depends on the buyer's expectations regarding the spot price $\tilde{p}_{2}$. Therefore, advance buyers will never pay more than $\tilde{p}_{2}$ without capacity constraints. However, as we show later, advance buyers may pay more than $\tilde{p}_{2}$ when capacity constraints are present.

Note that it is easy to generalize Theorem 2 to distributions having more than two states $(H, L)$. Consider a general density function $f(r)$ for buyer valuations where $L \leq r \leq H$. We find the maximum advance price (buyers will pay) by equating

$$
E S A=\int_{L}^{H} r f(r) d r-p_{1}
$$

with

$$
E S W=\int_{\tilde{p}_{2}}^{H}\left(r-\tilde{p_{2}}\right) f(r) d r
$$

So that

$$
\int_{\tilde{p}_{2}}^{H}\left(r-\tilde{p}_{2}\right) f(r) d r=\int_{L}^{H} r f(r) d r-p_{1},
$$

which is equivalent to

$$
\begin{aligned}
& \int_{L}^{H}\left(r-\tilde{p}_{2}\right) f(r) d r-\int_{L}^{\tilde{p}_{2}}\left(r-\tilde{p}_{2}\right) f(r) d r \\
& \quad=\int_{L}^{H} r f(r) d r-p_{1} .
\end{aligned}
$$

Solving for $p_{1}$, we obtain

$$
p_{1}^{\max }=\tilde{p}_{2}-\int_{L}^{\tilde{p}_{2}}\left(\tilde{p}_{2}-r\right) f(r) d r
$$

for a general distribution.

Also note that higher expected spot prices can increase the amount buyers will pay in the advance pe- riod. Hence, sellers may want to overstate spot prices in Period 1 to raise $\tilde{p}_{2}$ and get buyers to pay more in the advance period. The literature considers these announcements as credible only when the announcements are consistent with seller incentives. We subsequently analyze the problem with rational consumers (Coase 1972) who understand that, in Period 2, sellers will adopt the spot price that maximizes spot profits. Hence, buyers expect $\tilde{p}_{2}=p_{2}^{*}$. The issue of ex ante credibility arises in many other contexts where the assumption of naïve buyers is unacceptable (Padmanabhan et al. 1997, Sobel and Takahashi 1983). Beyond the basic Shugan-Xie model, there are other ways to ensure credibility. Later, we discuss capacity constraints as another method of ensuring credibility. We also discuss possible exogenous credibility due to reputation effects or other factors that allow sellers to credibly commit to their preannounced spot prices.

\subsection{Seller Strategy in the Advance Period}

We now compare profits from advance selling with spot-only strategies. Let the profits from advance selling and spot-only selling strategies be $\pi_{A}\left(p_{1}, p_{2}\right)$ and $\pi_{S}\left(p_{2}\right)$, respectively. For example, with an advanced price of $p_{1}^{\max }$, the profits from advance selling and spot-only selling are given in Equations (1) and (2):

$$
\begin{gathered}
\pi_{A}\left(p_{1}^{\max }, p_{2}\right)= \begin{cases}{[E V-c] N+q(H-c) N} \\
(L-c) 2 N & \text { if } p_{2}=L,\end{cases} \\
\pi_{S}\left(p_{2}\right)= \begin{cases}q(H-c) 2 N & \text { if } p_{2}=H \\
(L-c) 2 N & \text { if } p_{2}=L .\end{cases}
\end{gathered}
$$

Theorem 3 provides the conditions under which advance-selling profits exceed spot-only profits.

Theorem 3 (Basic Model-Optimal Seller Strategy in Periods 1 and 2). Let $c=(L-q H) /(1-q)$, then Table 1 provides the optimal seller strategies.

We see that when $L>c \geq \underline{c}$, the optimal strategy is to sell to all early arrivals at a price discounted to the spot price, i.e., $p_{1}^{*}=E V<p_{2}^{*}=H$. Advance selling increases profits by increasing sales by $(1-q) N$. These sales come from buyers who will later be in an unfavorable state and will not buy at a high spot 
Table 1 Optimal Advance Selling Strategies (Unlimited Capacity)

\begin{tabular}{|c|c|c|}
\hline Condition & Optimal Strategy & Conclusion About Profits \\
\hline $\begin{array}{l}\text { Small marginal cost } \\
\qquad(c<\underline{c})\end{array}$ & $\begin{array}{l}\text { Advance and spot sell } \\
\text { at a low price; or only } \\
\text { spot sell at a low } \\
\text { price. } p_{1}^{*}=p_{2}^{*}=L\end{array}$ & $\begin{array}{l}\text { Advance selling has the } \\
\text { same profits as spot- } \\
\text { only selling. }\end{array}$ \\
\hline $\begin{array}{l}\text { Medium marginal } \\
\qquad \operatorname{cost}(L>c>\underline{c})\end{array}$ & $\begin{array}{l}\text { Advance sell at a dis- } \\
\text { count to the spot } \\
\text { price. } p_{1}^{*}=E V<p_{2}^{*} \\
=H\end{array}$ & $\begin{array}{l}\text { Advance selling has } \\
\text { greater profits than } \\
\text { spot-only selling }\end{array}$ \\
\hline $\begin{array}{l}\text { Large marginal cost } \\
\qquad(c \geq L)\end{array}$ & $\begin{array}{l}\text { Spot sell at a high price; } \\
\text { no advance sales. } p_{2}^{*} \\
=H\end{array}$ & $\begin{array}{l}\text { Advance selling offers } \\
\text { no advantage. }\end{array}$ \\
\hline
\end{tabular}

price. However, when $c<\underline{c}$ the optimal spot price is low, which induces sales from all buyers, so advance selling has no advantage. When costs are too high ( $c$ $>L$ ) then there is no profit from selling to buyers in low states. Selling to these buyers with a discounted advance price is, therefore, not profitable. We conclude that in the basic model advance selling improves profits when both $c \geq \underline{c}$ and $L>c$.

We refer to the first condition $(c \geq \underline{c})$ as the credibility condition. This condition ensures that $p_{2}^{*}=H$. Advance selling only improves profits when buyers are willing to advance buy at a price greater than $L$. To advance sell at prices greater than $L$, buyers must believe that the spot price is also greater than $L$, as shown in Theorem 2. Otherwise, $p_{1}^{\max }=L$ and $\pi_{A}\left(p_{1}^{\max }, L\right)=\pi_{S}(L)$. In the basic model, the only way to achieve this credibility is for $c \geq c$, which ensures the actual spot price is greater than $L$, i.e., $p_{2}^{*}=H$. When $c<\underline{c}$, the credibility condition fails and the optimal spot price is $L$. Later, we discuss other means for establishing credibility, including capacity constraints.

We refer to the second condition $(L>c)$ as the profitability condition. This condition ensures that costs are sufficiently low so that serving customers in unfavorable states produces some profits. Advance selling increases profits when it allows advance sales to buyers who would be in unfavorable states later and would not purchase under a spot-only selling strategy. Selling to those buyers, however, is unprofitable when they value the product/service less than its cost. When costs are too high, $c \geq L$, the profitability condition fails, and advance selling provides no greater profits than spot selling at $H$.

As seen in the basic model, both the credibility and profitability conditions can thus be expressed as constraints on marginal cost, $L>c \geq \underline{c}$. Note that the basic model does not require early arrivals to exhibit greater price sensitivity than later arrivals or that capacity constraints be binding. The early arrivals and the later arrivals can have the same valuations. Therefore, the profit potential of advance selling is fundamental to a degree of buyer uncertainty and a range of seller's costs. Specific industry characteristics such as capacity constraints are unnecessary. The profit potential of advance-selling strategy is, therefore, far more general than previously realized. In the next section, we examine that profit potential and the impact of advance selling on social welfare.

\section{Profit and Social Welfare Implications}

\subsection{Profit Advantage of Advance Selling}

We now explore how much advance selling can improve profits compared with the best spot-only strategy. Theorem 4 shows the profit advantage of advance selling.

Theorem 4 (Profits Advantage). When $L>c \geq c$ and $\underline{c}=(L-q H) /(1-q)$, advance selling increases profits by $(1-q)(L-c) N$. Advance selling increases profits from early arrivals by $100(1-q)(L-c) /[q(H-c)]$ percent, which is maximized when $c=c$, where profits increase by 100(1-q) percent.

For example, when $N=10, L=2, H=11, c=1$, $q=0.1$, the advantage of advance selling is $(1-q)(L$ $-c) N=9$, and advance selling increases profits by $90 \%$.

Advance selling improves profits by $(1-q)(L-$ c) $N$. This improvement increases as the favorable-state probability $(q)$ decreases, the cost $(c)$ decreases, and the unfavorable-state valuation $(L)$ increases. However, the condition $c \geq \underline{c}=(L-q H) /(1-q)$ constrains all three variables. As Theorem 4 states, the improve- 
ment from advance selling is maximized when the condition exactly holds, i.e., $c=\underline{c}$, where profits from early arrivals increase by $100(1-q)$ percent. As $q$ approaches zero, advance-selling profits approach twice the profits from optimal spot selling to early arrivals.

\subsection{Social Welfare}

This section examines the impact of advance selling on social welfare. With a medium cost, i.e., $L>c \geq$ $c$, Theorem 3 states that sellers can improve profits by selling to all Period 1 arrivals at $p_{1}=E V$, where $E V=q H+(1-q) L$. Without advance selling, a fraction $q$ of the advance buyers will be in favorable states and spot buy at $p_{2}=H$. Others would not spot buy. The former group gains $q(H-E V)=q(1-q)(H-$ $L$ ) from advance buying, while the latter group loses $(1-q)(L-E R P)=-q(1-q)(H-L)$. Thus, the aggregate change in buyer surplus caused by advance selling is zero. Advance selling does not reduce buyers' total surplus because it simply shifts surplus from some buyers (those in unfavorable states) to other buyers (those in favorable states). While individual buyers may be better or worse off, the aggregate surplus remains the same. Since advance selling increases seller profits (see Theorem 4) without any loss in buyer surplus, it can increase social welfare. This leads to Theorem 5 .

Theorem 5 (Social Welfare). In the basic model, given midrange marginal cost (i.e., $L>c \geq c$ ) and a homogeneous market, advance selling has no impact on aggregate consumer surplus. Advance selling increases social welfare by $(1-q)(L-c) N$.

Theorem 5 states the welfare implications of the basic model, where the market is homogeneous, i.e., buyers have the same valuations and probabilities. Hence, seller profits do not improve at the expense of buyers. Sellers' profits improve because more buyers are able to purchase.

\section{Capacity Constraints}

We now examine the impact of capacity constraints on advance selling. We first consider the impact on buyer behavior and, subsequently, the impact on the seller's optimal strategy.

\subsection{Impact of Capacity Constraints on Buyers}

Let $T$ denote the seller's capacity. The basic model results when $T \geq 2 N$; this section considers binding capacity, i.e., $T<2 N$. Following the yield management literature, we allow sellers to limit advance sales to $S_{1}$, where $S_{1} \leq T$. This limit allows sellers to reserve $T-S_{1}$ capacity for sales in Period 2.

With limits on capacity, buyers may try to purchase but not succeed. For example, given an advance price not exceeding buyer expected valuations (i.e., $p_{1} \leq E V$, and a high spot price $p_{2}=H$ ), all buyers will attempt to advance purchase. So, the probability of obtaining capacity in Period 1 is $S_{1} / N$.

Buyers who try and fail to advance purchase enter the spot period. Depending on the spot price and their consumption state, they may try to spot purchase and compete for capacity with Period 2 arrivals. In the case in which $S_{1}$ buyers advance purchase and $p_{2}=H$, then $q\left(2 N-S_{1}\right)$ buyers will attempt to purchase in Period 2. The Period 2 capacity is $T-S_{1}$. When there is insufficient capacity for all buyers to spot purchase, i.e., $T-S_{1} \leq q\left(2 N-S_{1}\right)$, then the probability of obtaining capacity in Period 2 is ( $T$ $\left.S_{1}\right) / q\left(2 N-S_{1}\right)$. Theorem 6 follows.

Theorem 6 (Buyer Behavior with Capactty ConSTRAINTS). When the capacity constraint is binding, i.e., $T$ $<2 N$, and sellers limit Period 1 sales to $S_{1}$, where $0 \leq S_{1}$ $\leq \min \{N, T\}$, then the maximum price inducing an advance purchase is:

$$
p_{1}^{\max }= \begin{cases}E V & \text { if } p_{2}=H \\ L+q(H-L)\left[1-\lambda_{2}\right] & \text { if } p_{2}=L,\end{cases}
$$

where $\lambda_{2}=\left(\mathrm{T}-\mathrm{S}_{1}\right) /\left(2 \mathrm{~N}-\mathrm{S}_{1}\right)$ is the buyer's probability of getting capacity in Period 2 given $p_{2}=L$.

Theorem 6 shows that when spot prices are high, then buyers will pay $p_{1}^{\max }=E V$ in the advance period independent of expected capacity in the consumption period. Expected capacity is irrelevant because high spot prices eliminate both buyers' surplus from consumption and the opportunity cost associated with unavailable capacity. Buyers are indifferent between 
buying and being unable to buy given unavailable capacity.

Low spot prices $\left(p_{2}=L\right)$ produce a different consequence. Here, with probability $q$, buyers will have a high valuation and a positive surplus $(H-L)$. In that event, there is a probability $\left[1-\lambda_{2}\right]$ that the buyer will lose this positive surplus. The buyer is willing to pay a premium of at most $q(H-L)\left[1-\lambda_{2}\right]$ in the advance period. The premium represents the expected lost surplus when both capacity is unavailable and the buyer has a high valuation. When the probability of available capacity is zero $\lambda_{2}=0$, then the buyer will pay at most $E V$ in Period 1 because spot buying is not an option. When the probability of available capacity is certain $\lambda_{2}=1$, then the buyer will pay at most $L$ in Period 1 because spot buying is always possible at the spot price of $p_{2}=L$.

It might appear that the premium over the spot price that buyers pay in Period 1 is a risk premium and a form of insurance. This interpretation is incorrect. Risk-neutral and risk-seeing buyers will still pay a premium. Advance purchases do more than guarantee capacity. Remember that guaranteed capacity has no benefit when $p_{2}=H$. Advance buying allows buyers to secure the surplus that results when buyers have high valuations. Advance buying at prices below $p_{1}=q(H-L)\left[1-\lambda_{2}\right]$ simply provides a higher expected value than spot buying at $p_{2}=L$ with probability $\lambda_{2}$.

Hence, Theorem 6 shows that limited capacity can impact Period 1 buyer behavior when spot prices are low but has no impact on Period 1 buyer behavior when spot prices are high.

\subsection{Impact of Capacity Constraints on Sellers}

We now consider how capacity constraints impact the seller's optimal strategy. As noted earlier, sellers can limit first-period sales to $S_{1}$. In the special case in which $S_{1}=N$, there is no limit on first-period sales, and all first-period arrivals can advance buy if they choose to do so. Theorem 7 provides optimal seller strategies.

Theorem 7 (Impact of Capacity Constraints on SelleR Strategy). Let $\hat{p}_{1}=L+q(H-L)\left[1-\lambda_{2}\right], \hat{S}=(T$ $-2 q N) /(1-q)$, and $\lambda_{2}=\left(T-S_{1}\right) /\left(2 N-S_{1}\right)$, then
Table 2 Optimal Advance Selling Strategy with Capacity Constraints

\begin{tabular}{|c|c|}
\hline Capacity Condition & Optimal Strategy \\
\hline $\begin{array}{l}\text { Very small capacity: } \\
\qquad T \leq 2 q N\end{array}$ & $\begin{array}{l}p_{2}^{*}=H, \quad S_{1}=0 \\
\text { No advance sales }\end{array}$ \\
\hline $\begin{array}{l}\text { Small capacity: } \\
\qquad 2 q N<T<(1+q) N\end{array}$ & $\begin{array}{c}p_{1}^{*}=E V<p_{2}^{*}=H, \quad S_{1}=\hat{S} \\
\text { DISCOUNT Advance selling- } \\
\text { limit advance sales }\end{array}$ \\
\hline $\begin{array}{l}\text { Medium capacity: } \\
\qquad(1+q) N \leq T \leq\left[1+\left(\frac{H-c}{L-c}\right) q\right] N\end{array}$ & $\begin{array}{r}p_{1}^{*}=E V<p_{2}^{*}=H, \quad S_{1}=N, \\
\text { DISCOUNT Advance selling- } \\
\text { No limit on advance sales }\end{array}$ \\
\hline $\begin{array}{l}\text { Large capacity: } \\
{\left[1+\left(\frac{H-c}{L-C}\right) q\right] N<T<2 N}\end{array}$ & $\begin{array}{r}p_{1}^{*}=\hat{p}_{1}>p_{2}^{*}=L, \quad S_{1}=N \\
\text { PREMIUM Advance selling- } \\
\text { NO limit on advance sales }\end{array}$ \\
\hline
\end{tabular}

1. Advance selling improves profits in the bold cells.

2. The large-capacity case is only applicable when $[(H-c) /(L-c)] q<1$, or equivalently, $c<\underline{c}=(L-q H) /(1-q)$.

Table 2 provides the optimal seller strategies with capacity constraints when $c<L$. The optimal seller strategy when $c \geq L$ is spot selling only at a high spot price, $p_{2}^{*}=H, S_{1}$ $=0$.

\subsection{Conclusion on Capacity Constraints}

Theorem 7 reveals several interesting findings. First, we find that with limited capacity the profitability condition (i.e., $L>c$ ) is still required, but the credibility condition (i.e., $c \geq \underline{c}$ ) is no longer required for advance selling to improve profits. Recall that without capacity constraints, a large marginal cost (i.e., $c$ $\geq \underline{c}$ ) was necessary to make a high spot price credible. Without that high spot price, buyers would not advance purchase. Theorem 7 reveals that a constraint on capacity (i.e., $2 q N<T<2 N$ ) can make advance selling profitable. When capacity is not large (i.e., $T$ $\leq\{1+[(H-c) /(L-c)] q\} N)$, constrained capacity creates credibility by making a high spot price optimal. With large but limited capacity (i.e., $\{1+[(H-$ c) $/(L-c)] q\} N<T<2 N)$, we find premium advance selling becomes optimal, which leads to our second implication.

A secondary interesting implication is that capacity constraints can allow sellers to charge a premium for 
advance purchases. Recall that without capacity constraints, buyers will pay no more in the advance period than the expected spot price. With unavailable capacity, however, there is an infinite spot price. Advance buyers must now consider both the spot price and the likelihood of unavailable capacity. When sellers choose a spot price of $p_{2}=L$, then the buyer is willing to advance purchase at a higher price than the spot price. Hence, it is possible to have advance prices that are higher than spot prices. Here, buyers advance purchase because the expected surplus is higher than waiting, given a probability of unavailable capacity. The premium paid in the advance period is not insurance because risk-neutral buyers still advance purchase.

Although premium advance selling is inconsistent with current strategies employed by the airline industry, that observation may depend on specific industry characteristics (i.e., late arrivals by price-insensitive business travelers). Theorem 7 reveals that other industries may find it profitable to adopt other advance-selling strategies, such as advance selling at higher than spot prices. In other travel businesses, for example, Dawe (2000) finds last-minute bargain travel opportunities for flexible travelers who are willing to accept only a chance of travel (i.e., a positive probability of unavailable capacity). Here, last-minute purchasers accept the possibility of unavailable capacity in exchange for a low spot price.

Another example occurs in retailing. ${ }^{3}$ A department store's seasonal inventory is analogous to a limited capacity because no additional quantity is available in a season. Preseason, buyers will purchase at a premium price because later buyers, who find themselves in need of an item, may find the item already sold (i.e., capacity becomes unavailable). Here, buyers advance purchase prior to the season to guarantee capacity, and advance selling becomes profitable. Risk aversion is not required and, therefore, this is not an example of insurance. This explanation of retail markdowns does not require retailer demand uncertainty but is consistent with that literature (Lazear 1986).

Finally, note that premium pricing is only optimal when $[(H-c) /(L-c)] q<1$, which is when the cred-

\footnotetext{
${ }^{3}$ We thank Brian Ratchford for this example.
}

ibility condition fails, i.e., $c<\underline{c}$. When credibility holds, the large-capacity condition in Table 2 is never satisfied and the medium-capacity case is applicable for all capacities larger than $(1+q) N$. Hence, premium advance pricing increases profits only when high spot prices are not credible and when capacity is limited $(T<2 N)$. Otherwise, a high spot price is best.

A third implication of Theorem 7 is consistent with Desiraju's and Shugan's (1999) findings. We also find yield management systems that limit advance sales are only necessary under specific conditions. We find that limiting capacity $(\hat{S}=(T-2 q N) /(1-q))$ with YMS is only necessary for a range of capacities such that $0<\hat{S}<N$, which corresponds to the condition $2 q N<T<(1+q) N$. Outside of that range, sellers have no need for YMS. When $\hat{S} \geq N$, then $T \geq(1+$ q) $N$ and advance sales should occur without limit. When $\hat{S} \leq 0$, then $T \leq 2 q N$ and no advance sales should be made, which leads to the fourth implication.

A fourth implication of Theorem 7 is that, although limited capacity can create the ability to advance sell, it can also decrease the incentive to advance sell. Recall that with unlimited capacity the superiority of advance selling comes from greater volume sales, i.e., sales of $N$ rather than sales of $q N$. Capacity constraints can reduce the incentive to increase demand because with less capacity to sell, there can be less need to increase sales. When $T \leq 2 q N$, then there is no additional profit from advance selling.

Finally, for industries that exhibit extreme seasonality (Krider and Weinberg 1998, Radas and Shugan 1998a), our findings suggest that advance-selling strategies should change during seasons when capacity is more limited.

\section{Other Factors}

The following three subsections discuss how three other factors may impact the optimal advance-selling strategy. We examine the impact of (1) buyer risk aversion, (2) seller exogenous credibility, and (3) partial refunds by the seller. Although a complete analysis awaits future research, we provide specific advance-selling strategies for special cases. 
XIE AND SHUGAN

When and How to Advance Sell

Table 3 Impact of Risk Aversion

\begin{tabular}{|c|c|c|c|}
\hline & $U_{j}(x)$ & $p_{1}^{\max }$ & $\begin{array}{c}\text { Numerical Solution }(H=10, L=5, \\
q=0.5, w=15, k=5, c=1)\end{array}$ \\
\hline Case 1 (risk-neutral) & $\begin{cases}k+x & \text { if } j=F \\
x & \text { otherwise }\end{cases}$ & $q H+(1-q) L$ & $p_{1}^{\max }=7.5$ \\
\hline Case 2 (risk-averse) & $\begin{cases}k+\sqrt{x} & \text { if } j=F \\
\sqrt{x} & \text { otherwise }\end{cases}$ & $w-[q \sqrt{w-H}+(1-q) \sqrt{w-L}]^{2}$ & $p_{1}^{\max }=7.7$ \\
\hline Case 3 (risk-averse) & $\begin{cases}k+\log (x) & \text { if } j=F \\
\log (x) & \text { otherwise }\end{cases}$ & $w-\exp [q \log (w-H)+(1-q) \log (w-L)]$ & $p_{1}^{\max }=7.9$ \\
\hline Case 4 (risk-averse) & $\begin{cases}\sqrt{k+x} & \text { if } j=F \\
\sqrt{x} & \text { otherwise }\end{cases}$ & $\begin{array}{l}q\left(\sqrt{k+w-p_{1}^{\max }}\right)+(1-q) \sqrt{w-p_{1}^{\max }} \\
\quad=q(\sqrt{k+w-H})+(1-q) \sqrt{w-L}\end{array}$ & $p_{1}^{\max }=7.2$ \\
\hline
\end{tabular}

\subsection{Buyer Risk Aversion}

It might appear that the profit advantage from advance selling depends on risk neutrality. Buyer risk aversion could make advance purchasing less attractive because future valuations are uncertain. Sellers might need to make deeper discounts in the advance period, making advance selling less profitable. This section determines whether the profits from advance selling are a consequence of the risk-neutrality assumption.

The profits with advance selling are $\left(p_{1}^{\max }-c\right) N+$ $q(H-c) N$. We now examine the impact of buyer risk attitude on $p_{1}^{\max }$ because a larger $p_{1}^{\max }$ implies greater profits.

To proceed, we must define the buyer's utility for wealth (Savage 1954). Let $U_{j}(w-p)$ denote the buyer's utility given initial wealth $w$, a purchase price $p$, and consumption of the service in state $j$ where $j=$ $F, U$ for favorable and unfavorable, respectively. By definition, risk aversion implies that $U_{j}^{\prime \prime}(w)<0$. It follows that the expected utility from advance buying at price $p_{1}$ is $q U_{F}\left(w-p_{1}\right)+(1-q) U_{U}\left(w-p_{1}\right)$.

Now consider the expected utility of not advance buying when the spot price is $p_{2}=H$. If the buyer realizes a favorable state and purchases at $p_{2}=H$, then the buyer's utility is $U_{F}(w-H)$. If the buyer realizes an unfavorable state and does not purchase, then the buyer's utility is $U_{U}(w-L)$ because the utility from not consuming exactly equals the utility from consuming at price $L$. Hence, the expected util- ity of not advance buying becomes $q U_{F}(w-H)+(1$ - q) $U_{U}(w-L)$.

In the advance period, the buyer will pay no more than the price that equates the expected utility of advance buying and not. Hence, we find this maximum price $p_{1}^{\max }$ from the following equation:

$$
\begin{gathered}
q U_{F}\left(w-p_{1}^{\max }\right)+(1-q) U_{U}\left(w-p_{1}^{\max }\right) \\
=q U_{F}(w-H)+(1-q) U_{U}(w-L) .
\end{gathered}
$$

Table 3 provides the advance price $p_{1}^{\max }$ given four specific utility functions. The first function is risk neutral and the others are risk averse. For example, consider the special case in which $H=10, L=5, q$ $=1 / 2, W=15, k=5, c=1$. Then, the risk-neutral buyer will pay $p_{1}^{\max }=7.5$. We find that for two common utility functions (Case 2 and Case 3), risk aversion increases the profitability of advance selling because the buyers will pay a higher advance price (i.e., $p_{1}^{\max }=7.7$ in Case 2 and $p_{1}^{\max }=7.9$ in Case 3). In general, however, it is not possible to determine whether the profits will be higher or lower for a risk averse buyer. It depends on whether the buyer is more afraid of not advance buying in a favorable state (paying $H$ rather than $p_{1}^{\max }$ ) or advance buying in an unfavorable state (paying $p_{1}^{\max }$ rather than not consuming). It also depends on how the utility function differs in favorable and unfavorable states. In Case 4, the profits are lower because $p_{1}^{\max }=7.2$. We conclude 
that the profit advantage of advance selling is not the result of risk neutrality. The impact of risk however, deserves future research.

\subsection{Sellers with Exogenous Credibility}

This section considers the situation in which buyers believe the spot price announced by the seller in Period 1 regardless of whether that spot price maximizes seller profits in Period 2.

The basic model equates buyers' expected spot prices to sellers' optimal spot prices because sellers are unable to credibly commit to their preannounced spot prices otherwise. This section examines situations where reputation effects or other factors allow sellers to credibly commit to their preannounced spot prices. For example, the Philadelphia Zoo offers 3-week-in-advance tickets at $\$ 10.50$ and gate tickets at $\$ 8.40$ everyday (www.phillyzoo.org/pz-gs10.htm). Hence, on each day the zoo would have a mix of buyers who paid advanced and spot prices. Because spot prices are observable, they are credible, regardless of whether those prices are sellers' optimal spot prices. Many services offer such discounted advance tickets and higher priced spot tickets simultaneously and routinely (e.g., Fetish Beach Ball (xorvia.org/ beachball.html), truck and car shows (familyevents. com/calendar/calendar.html), and bridal shows (elegantbridalshow.com/orderform.html). We refer to those sellers as "sellers with exogenous credibility."

Only sellers with exogenous credibility can implement an advance-selling strategy $\left\{p_{1}=p_{1}^{\max }, p_{2}=H\right\}$, even if the high spot price, $p_{2}=H$, produces less spot profits than $p_{2}=L$. Theorem 8 follows.

Theorem 8 (Optimal Pricing with Exogenous CrediBILITY). For sellers with exogenous credibility, advance selling earns greater profits than spot selling when and only

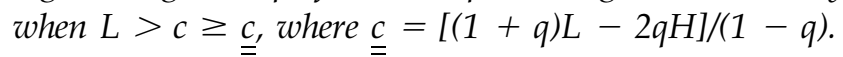

In the basic model (Theorem 3$)$, endogenous credibility requires $c \geq \underline{c}=(L-q H) /(1-q)$. With exogenous credibility, we now only require $c \geq \underline{c}$, where $\underline{c}<\underline{c}$. Hence, exogenous credibility provides a greater opportunity for advance selling. Without exogenous credibility, buyers will expect a low spot price (given $c<\underline{c}$ ) and will not advance purchase at $E V$ (à la The- orem 2). With it, buyers expect high spot prices despite seller incentives to have a low spot price. Exogenous credibility gives the seller the ability to announce a believable high spot price when $c \leq c \leq$ c. Then, the buyers advance purchase at $E V$ when $\underline{\underline{c}}$ $\leq c \leq c$, giving advance selling a profit advantage over only spot selling. Future research should investigate mechanisms for establishing exogenous credibility.

\subsection{Refunds}

It might appear that refunds destroy the advantage of advance selling because buyers no longer commit themselves to purchasing. Without that commitment, the ability of advance selling to increase sales is questionable. This section shows, despite lower sales with refunds, that advance selling with partial refunds can provide more profit than advance selling without them.

With partial refunds, buyers who advance purchase may later decide not to consume in exchange for a partial refund. Consider a partial refund $R>0$. We allow buyers to have three states: a favorable state, an unfavorable state, and a no-value state. Buyers in no-value states are unwilling to pay any positive price for the service and prefer partial refunds to consumption, i.e., they will consume without refunds and will take the refund, otherwise. Let buyer valuations in these states be $H, L$, and zero, respectively. The three states occur with probabilities $q_{H}, q_{L}$, and $q_{0}$, respectively.

First, consider the case without refunds. Remember, buyers advance purchase only when the expected surplus of advance buying (ESA) exceeds the expected surplus of waiting (ESW). Without refunds, ESA $=q_{H} H+q_{L} L-p_{1}$. Equation (4) provides ESW.

$$
E S W= \begin{cases}0 & \text { if } p_{2}^{*}=H \\ q_{H}(H-L) & \text { if } p_{2}^{*}=L .\end{cases}
$$

The maximum price inducing an advance purchase $\left(p_{1}^{\max }\right)$ sets $E S A=E S W$. See Equation (5):

$$
p_{1}^{\max }(\text { No Refund })= \begin{cases}q_{H} H+q_{L} L & \text { if } p_{2}^{*}=H \\ \left(q_{H}+q_{L}\right) L & \text { if } p_{2}^{*}=L .\end{cases}
$$

Now, consider the case with refunds. With a refund 
of $R \leq L$, buyers will take the refund rather than consuming in a no-value state. The expected surplus of waiting, ESW, is the same as without refunds. Buyers' expected surplus from advance purchasing is $E S A=$ $q_{H} H+q_{L} L+q_{0} R-p_{1}$. Thus, $p_{1}^{\max }$ is given by Equation (6).

$$
p_{1}^{\max }(\text { Refund })= \begin{cases}q_{H} H+q_{L} L+q_{0} R & \text { if } p_{2}^{*}=H \\ \left(q_{H}+q_{L}\right) L+q_{0} R & \text { if } p_{2}^{*}=L .\end{cases}
$$

Comparing the profit from only spot selling with that from advance selling with and without refunds, Theorem 9 follows.

THEOREM 9 (REFUNDS). When buyer valuations are $H, L$, and zero with probabilities $q_{H}, q_{L}$, and $q_{0}$, respectively, and $L>c \geq c$, where $\underline{c}=(L-q H) /(1-q)$, advance selling with a refund, $L \geq R>0$, earns higher profits than advance selling without refunds and earns higher profits than only spot selling. Offering a refund increases the profit from advance selling by $q_{0} c$.

Theorem 9 shows that advance selling with partial refunds is not only more profitable than spot-only selling, but also more profitable than advance selling without partial refunds (provided $L \geq R$ ). This finding may seem counterintuitive until we realize that partial refunds increase profits not by increasing revenues but by cost savings from not serving customers in no-value states. Given that buyers will pay more in the advance period when refunds are available, the higher advance price compensates for the actual cost of the refund (i.e., provided that $L \geq R$ ). Theorem 9 shows that offering refunds increases the profit of advance selling by $q_{0} c$. The higher the buyers' probability of having a no-value state and the larger the marginal cost, the more the seller benefits from offering partial refunds. Other situations with multiple states and buyer heterogeneity deserve future research. Efficiency should also be explored (Fruchter and Gerstner 1999).

\section{Conclusions}

Although advance selling had the potential to be an important marketing tool, problems in implementation have limited its usefulness. As new technologies (e.g., electronic tickets, smart cards, Web-based prepayments) are overcoming those impediments, we expect that small service providers will soon enjoy the sophisticated pricing strategies previously employed only by the largest companies. Small sellers can now advance sell on a website, allowing buyers to buy services in advance without physically visiting the service provider. As these events unfold, more academic attention to advance selling is vital. This paper tries to help exploit these emerging technologies by studying when and how advance-selling strategies are optimal.

This paper significantly generalizes the Shugan-Xie model (Shugan and Xie 2000) to provide two general findings and several specific findings.

\subsection{General Findings}

First, as Figure 1 shows, advance-selling tools are more applicable than previously thought. Buyers are nearly always uncertain about their future valuation of most services, and that uncertainty alone can make advance selling profitable. Unlike most previous research, we show advance selling can be profitable without industry-specific factors such as capacity constraints, buyer risk aversion, late arrivals by price-insensitive customers, buyer convenience, or restrictions on advance sales. Advance selling can help service providers in any industry in which buyers have uncertainty about their future valuations for a service (provided announced spot prices are credible and marginal costs are within a specified range). Advance selling can, depending on parameter values, double the profits from optimal spot-only selling to early arrivals. We also show that advance selling has no impact on consumer surplus in markets with homogeneous consumers and no capacity constraints (i.e., the basic model). Therefore, advance selling can increase social welfare because seller profits increase.

Second, unlike most previous research, we show that advance selling at a premium is sometimes optimal. Although advance selling should often be at a discount on the spot price (see Figure 1), we find that advance selling at a premium is optimal with large but limited capacity and marginal costs that are not large. This strategy benefits sellers who are unable to 
credibly establish a high spot price, but can credibly establish a lack of capacity. No prior research has suggested this strategy because that research relies on a negative correlation between the time of arrival and price sensitivity. Without that assumption, premium advance pricing is sometimes optimal. Figure 1 also reveals that advance sales should be limited when capacity is insufficient to satisfy spot demand (at a high spot price) but marginal costs are sufficiently small so that discounted advance prices are still profitable. Buyers advance purchase to guarantee capacity given high future valuations because the expected surplus makes it worthwhile. Although this appears to be insurance, it is not because risk-neutral buyers still advance purchase.

\subsection{Specific Findings}

Advance selling increases profits when marginal costs are sufficiently large or capacity is sufficiently small. The reason is that buyers advance purchase only when they expect an advantage from doing so. There are two sources of advantage. First, sellers can credibly convince buyers that the advance price is at a discount to the spot price. Large marginal costs or limited capacity can provide that credibility. Second, buyers may advance buy to avoid the possibility of unavailable capacity in the spot period. Limited capacity creates that possibility.

The advantage from advance selling also requires sufficiently low costs. The reason is that advance selling increases sales by selling to buyers who would not spot purchase because they find themselves in an unfavorable state in the spot period. The seller induces advance purchases with a discounted price. Consequently, the seller enjoys additional sales, albeit at a lower profit contribution. When costs are too high, the required discount results in no profit advantage.

When capacity constraints are binding, advance selling can work in two very different ways. First, with limited capacity, advance selling can increase total unit sales to a level higher than that from only spot selling at a high spot price. (When capacity is very small, however, there is insufficient capacity to increase sales.) Second, when capacity is sufficiently large, but not unlimited, advance selling can increase profit margins by selling at an advance price higher than a low spot price.

Consistent with Desiraju and Shugan (1999), we find that limiting advance sales can be profitable but only under restrictive conditions (i.e., a range of capacities and a range of marginal costs). It is best to limit advance sales to reserve capacity for spot sales to buyers in favorable states when sellers would be left with insufficient capacity to sell to all late arrivals at a high spot price.

It is possible that other factors such as buyer risk aversion, exogenous credibility, and partial refunds can increase the profitability of advance selling. However, there are often other conditions required to insure that increase.

\subsection{Future Research}

Despite our analyses of advance selling in this paper, we leave many interesting areas for future research. Our model of advance selling can be extended in different directions and integrated with other research. For example, future research might integrate buyer uncertainty about future valuations into the existing literatures on bundling strategies (Ansari et al. 1996), the need for variety (Kahn and Lehmann 1991), delaying purchases (Greenleaf and Lehmann 1995), learning the demand function (Lazear 1986), and in channels situations (Padmanabhan and Png 1995).

We provided only a partial analysis of buyer surplus, buyer risk aversion, refunds, and exogenous credibility. Future research is clearly needed on these topics. For example, how can sellers create exogenous credibility, and what factors cause buyers to believe price announcements for future periods?

The theory of advance selling in this paper can be extended to include factors present in a variety of contexts. For example, future research can consider our model in conjunction with bundling across time and across services. This topic is relevant to sellers such as health clubs, country clubs, and other organizations that sell memberships. It is possible that advance bundling provides more profit than selling bundles at the time of consumption.

Other situations to consider include situations in which seller marginal costs depend on the state of the 
buyer (e.g., insurance or financial loans), situations in which buyer psychology changes as the spot period approaches (e.g., attention to different attributes might change), and situations in which buyers can purchase an assortment of services to be consumed at different times (e.g., a vacation package with vouchers for future unscheduled activities). Situations in which the sellers have different degrees of control over the time of consumption should be studied. In general, there has been little research about when buyer valuations have greater uncertainty and what creates that uncertainty. Situations in which buyer uncertainty about future valuations changes dynamically across time should also be studied.

Finally, rich areas of future research include how far in advance the sellers should advance sell, how to set multiple advance prices in multiple periods preceding consumption, how to advance sell in competitive environments, how to advertise advance sales, which services to advance sell in a service line, and how to advance sell bundles of services. Perhaps advance selling can be used to "lock out" competition, selling to buyers before they can spot purchase from competitors.

\section{Acknowledgments}

Authors are listed randomly; both authors contributed equally to the paper. The authors would like to thank Barton Weitz, David Sappington, Joseph Alba, Tracy Lewis, Brian Ratchford, Steven Slutsky, Lyle Brenner, Richard Lutz, Joel Cohen, Richard Romano, Carol West and the participants of the University of Florida Winter Research Retreat for their many helpful comments.

\section{Appendix}

Proof of Theorem 1 (Basic Model-Optimal Seller Strategy in PeRIOD 2). Let $N_{2}\left(p_{1}\right)$ denote the number of consumers in Period 2. $N_{2}\left(p_{1}\right)$ is a function of the Period 1 price because it depends on the number of buyers who have not purchased in Period 1. Let $\pi_{2}\left(p_{2}\right)$ denote seller Period 2 profits. Then,

$$
\pi_{2}\left(p_{2}\right)= \begin{cases}0 & \text { if } p_{2}>H \\ \left(p_{2}-c\right) q N_{2}\left(p_{1}\right) & \text { if } L<p_{2} \leq H \\ \left(p_{2}-c\right) N_{2}\left(p_{1}\right) & \text { if } p_{2} \leq L .\end{cases}
$$

Note that with homogeneous buyers, $N_{2}\left(p_{1}\right)$ will be either $N$ (Period 1 arrivals advance buy) or $2 N$ (Period 1 arrivals wait). In both cases, $\pi_{2}\left(p_{2}>H\right)<\pi_{2}\left(L<p_{2}<H\right)<\pi_{2}\left(p_{2}=H\right)$ and $\pi_{2}\left(p_{2}<L\right)$ $<\pi_{2}\left(p_{2}=L\right)$. Further, in both cases, $\pi_{2}\left(p_{2}=H\right) \geq \pi_{2}\left(p_{2}=L\right)$ if $q(H-c) \geq(L-c)$. Thus, in both cases, $p_{2}^{*}=H$ when $c \geq \underline{c}=(L$ $-q H) /(1-q)$, and $p_{2}^{*}=L$, otherwise. Theorem 1 follows.

Proof of Theorem 2 (Basic Model-Buyer Behavior in Period 1). Period 1 arrivals advance buy if $E S A-E S W \geq 0$, where $E S A=q H$ $+(1-q) L-p_{1}$ and

$$
E S W= \begin{cases}0 & \text { if } \tilde{p}_{2}>H \\ q\left(H-\tilde{p}_{2}\right) & \text { if } L<\tilde{p}_{2} \leq H \\ q H+(1-q) L-\tilde{p}_{2} & \text { if } \tilde{p}_{2} \leq L .\end{cases}
$$

Subtracting,

$$
E S A-E S W= \begin{cases}q H+(1-q) L-p_{1} & \text { if } \tilde{p}_{2}>H \\ q \tilde{p}_{2}+(1-q) L-p_{1} & \text { if } L<\tilde{p}_{2} \leq H \\ \tilde{p}_{2}-p_{1} & \text { if } \tilde{p}_{2} \leq L .\end{cases}
$$

Rearranging, $E S A-E S W \geq 0$ if

$$
p_{1} \leq p_{1}^{\max }= \begin{cases}q H+(1-q) L & \text { if } \tilde{p}_{2}>H \\ q \tilde{p}_{2}+(1-q) L & \text { if } L<\tilde{p}_{2} \leq H \\ \tilde{p}_{2} & \text { if } \tilde{p}_{2} \leq L,\end{cases}
$$

where $E V=q H+(1-q) L$. Theorem 2 follows.

Proof of Threorem 3 (Basic Model-Optimal Seller Strategy in Periods 1 and 2). Find the Optimal Period 1 Price. The profits of advance selling at $p_{1}$ is given by

$$
\pi_{A}\left(p_{1}, p_{2}^{*}\right)=\left\{\begin{array}{lll}
q(H-c) 2 N & \text { if } p_{2}^{*}=H, & p_{1}>p_{1}^{\max } \\
{\left[p_{1}-c\right] N+q(H-c) N} & \text { if } p_{2}^{*}=H, & p_{1} \leq p_{1}^{\max } \\
(L-c) 2 N & \text { if } p_{2}^{*}=L, & p_{1}>p_{1}^{\max } \\
{\left[p_{1}-c\right] N+(L-c) N} & \text { if } p_{2}^{*}=L, & p_{1} \leq p_{1}^{\max } .
\end{array}\right.
$$

Clearly, $\pi_{A}\left(p_{1}=p_{1}^{\max }, p_{2}^{*}\right)>\pi_{A}\left(p_{1}<p_{1}^{\max }, p_{2}^{*}\right), \pi_{A}\left(p_{1}=p_{1}^{\max }, p_{2}^{*}\right.$ $=L)=\pi_{A}\left(p_{1}>p_{1}^{\max }, p_{2}^{*}=L\right)$, and $\pi_{A}\left(p_{1}=p_{1}^{\max }, p_{2}^{*}=H\right)-\pi_{A}\left(p_{1}\right.$ $\left.>p_{1}^{\max }, p_{2}^{*}=H\right)=[q H+(1-q) L-c] N-q(H-c) N=(1-q)(L$ $-c) N>0$. Thus, $p_{1}^{*}=p_{1}^{\max }$.

Compare Advance Selling with Spot-Only Strategies. The profits from advance and spot-only selling are

$$
\pi_{A}\left(p_{1}^{*}, p_{2}^{*}\right)= \begin{cases}{[q H+(1-q) L-c] N+q(H-c) N} & \text { if } p_{2}^{*}=H \\ (L-c) 2 N & \text { if } p_{2}^{*}=L,\end{cases}
$$

and

$$
\pi_{S}\left(p_{2}^{*}\right)= \begin{cases}q(H-c) 2 N & \text { if } p_{2}^{*}=H \\ (L-c) 2 N & \text { if } p_{2}^{*}=L .\end{cases}
$$

Subtracting,

$$
\pi_{A}\left(p_{1}^{*}, p_{2}^{*}\right)-\pi_{S}\left(p_{2}^{*}\right)= \begin{cases}(1-q)(L-c) & \text { if } p_{2}^{*}=H \\ 0 & \text { if } p_{2}^{*}=L .\end{cases}
$$

Thus, $\pi_{A}\left(p_{1}^{*}, p_{2}^{*}\right)>\pi_{S}\left(p_{2}^{*}\right)$, if two conditions hold: (1) $p_{2}^{*}=H$ and 
(2) $L>c$. The first condition holds if $c \geq \underline{c}=(L-q H) /(1-q)$. Theorem 3 follows.

Proof of Theorem 4 (Profits Advantage). Assume $L>c \geq c$. The profit advantage of advance selling from Period 1 arrivals is $[q H+$ $(1-q) L-c]-[q H-c]=(1-q)(L-c)$. Expressing the profit advantage as a fraction of the spot-only profits yields

$$
\frac{(1-q)(L-c)}{q(H-c)}=\frac{\left(\frac{1}{q}-1\right)(L-c)}{H-c} .
$$

This ratio is maximized when $q$ takes it smallest value. Given the credibility condition, $q$ can be no smaller than $q^{*}=(L-c) /(H-$ c). When $q=q^{*}$, the profit advantge of advance selling is $(1-q)(L$ $-c) / q(H-c)=(1-q)$. Theorem 4 follows.

Proof of Theorem 5 (Social Welfare). Assume $L>c \geq \underline{c}$. Under the optimal advance-selling strategy, seller surplus from Period 1 arrivals is $S S_{A}=(E V-c) N$. Consumer surplus is $C S_{A}=[q(H-$ $E V)+(1-q)(L-E V)] N=0$. Social welfare is $S W_{A}=S S_{A}+C S_{A}$ $=(E V-c) N$. Under the optimal spot-selling strategy, seller surplus from Period 1 arrivals is $S S_{S}=q(H-c) N$, consumer surplus is $C S_{S}$ $=q(H-H) N=0$, and social welfare is $S W_{S}=S S_{S}+C S_{S}=q(H$ $-c) N$. Ergo, $S S_{A}-S S_{S}=(1-q)(L-c)>0, C S_{A}-C S_{S}=0, S W_{A}$ $-S W_{S}=(1-q)(L-c)>0$. Theorem 5 follows.

Proof of Theorem 6 (Buyer Behavior with Capacity Constraints). When sellers advance sell, a Period 1 arrival has two options: (1) try to advance purchase or (2) wait. The decisions are based on the expected surplus of the two options: the expected surplus from trying to advance buy (ESTA) and the expected surplus from waiting $(E S W)$. A Period 1 arrival will take option 1 if $E S T A \geq 0$ and $E S T A \geq E S W$.

Let $S_{1}$ denote the seller's limit on sales in Period $1,0 \leq S_{1} \leq$ $\min [N, T]$, and $\lambda_{t}$ denote the probability that a Period 1 arrival finds capacity in period $t$.

Option 1: Try to Advance Purchase. With this option, the expected surplus depends on the spot price. Specifically,

$$
\text { ESTA }=\left\{\begin{array}{l}
\left(E V-p_{1}\right) \lambda_{1}+q\left(H-p_{2}\right) \lambda_{2}\left(1-\lambda_{1}\right)=\left(E V-p_{1}\right) \lambda_{1} \\
\quad \text { if } p_{2}=H \\
\left(E V-p_{1}\right) \lambda_{1}+\left(E V-p_{2}\right) \lambda_{2}\left(1-\lambda_{1}\right) \\
\quad=\left(E V-p_{1}\right) \lambda_{1}+(E V-L) \lambda_{2}\left(1-\lambda_{1}\right) \\
\quad \text { if } p_{2}=L .
\end{array}\right.
$$

Note, ESTA $\geq 0$ if $p_{1} \leq E V$.

Option 2: Wait. With this option, the expected surplus depends on the spot price. Specifically,

$$
E S W= \begin{cases}\lambda_{2} q\left(H-p_{2}\right)=0 & \text { if } p_{2}=H \\ \lambda_{2}\left(E V-p_{2}\right)=\lambda_{2}(E V-L) & \text { if } p_{2}=L .\end{cases}
$$

Comparison. Subtracting,

$$
E S T A-E S W= \begin{cases}\left(E V-p_{1}\right) \lambda_{1} & \text { if } p_{2}=H \\ \left(E V-p_{1}\right) \lambda_{1}-(E V-L) \lambda_{1} \lambda_{2} & \text { if } p_{2}=L .\end{cases}
$$

Rearranging, ESTA $\geq E S W$ if $p_{1} \leq p_{1}^{\max }$, where

$p_{1}^{\max }= \begin{cases}E V & \text { if } p_{2}=H \\ L+(E V-L)\left(1-\lambda_{2}\right)=L+q(H-L)\left(1-\lambda_{2}\right) & \text { if } p_{2}=L\end{cases}$

and

$$
\lambda_{2}=\frac{T-S_{1}}{2 N-S_{1}} .
$$

Theorem 6 follows.

Note that in Theorem 6 we do not assume possible cooperation among buyers. By allowing cooperation, we let $\lambda_{2}$ denote the probability of available capacity in Period 2 when all Period 1 arrivals try to advance buy. Let $\lambda_{2}^{\prime}$ denote the probability of available capacity in Period 2 when all Period 1 arrivals wait. Hence,

$$
E S T A=\left\{\begin{array}{l}
\left(E V-p_{1}\right) \lambda_{1}+q\left(H-p_{2}\right) \lambda_{2}\left(1-\lambda_{1}\right)=\left(E V-p_{1}\right) \lambda_{1} \\
\quad \text { if } p_{2}=H \\
\left(E V-p_{1}\right) \lambda_{1}+\left(E V-p_{2}\right) \lambda_{2}\left(1-\lambda_{1}\right) \\
=\left(E V-p_{1}\right) \lambda_{1}+(E V-L) \lambda_{2}\left(1-\lambda_{1}\right) \\
\quad \text { if } p_{2}=L,
\end{array}\right.
$$

and

$$
E S W= \begin{cases}\lambda_{2}^{\prime} q\left(H-p_{2}\right)=0 & \text { if } p_{2}=H \\ \lambda_{2}^{\prime}\left(E V-p_{2}\right)=\lambda_{2}^{\prime}(E V-L) & \text { if } p_{2}=L .\end{cases}
$$

Comparing,

$$
\begin{aligned}
& E S T A-E S W \\
& = \begin{cases}\left(E V-p_{1}\right) \lambda_{1} & \text { if } p_{2}=H \\
\left(E V-p_{1}\right) \lambda_{1}-(E V-L)\left[\lambda_{2}^{\prime}-\lambda_{2}\left(1-\lambda_{1}\right)\right] & \text { if } p_{2}=L .\end{cases}
\end{aligned}
$$

Rearranging, ESTA $\geq E S W$ if

$$
\begin{aligned}
p_{1} & \leq p_{1}^{\max } \\
& = \begin{cases}E V & \text { if } p_{2}=H \\
L+(E V-L)(1-\Lambda)=L+q(H-L)(1-\Lambda) & \text { if } p_{2}=L,\end{cases}
\end{aligned}
$$

where

$$
\Lambda=\frac{\lambda_{2}^{\prime}-\lambda_{2}\left(1-\lambda_{1}\right)}{\lambda_{1}}, \quad 1 \geq \Lambda \geq 0 .
$$

Hence, with capacity constraints, buyers are willing to pay a premium $(q(H-L)(1-\Lambda)>0)$ for advance purchase when the spot price is low. Hence, when buyers cooperate we get the same results as Theorem 6 (buyers will pay a premium when $p_{2}=L$ and $E V$ when $p_{2}=H$ ), only the probability is $\Lambda$ rather than $\lambda_{2}$.

Proof of Theorem 7 (Impact of Capacity Constraints on Seller Strat- 
EGY). With minor modifications, the proof of Theorem 1 shows there are two possible optimal spot strategies, $p_{2}=H$ and $p_{2}=L$. With minor modifications, the proof of Theorem 3 shows the optimal advance price is $p_{1}^{\max }$, which depends on $p_{2}$. Define $p_{1 H}^{\max }$ and $p_{1 L}^{\max }$ as the corresponding $p_{1}^{\max }$ for each strategy. From Theorem 6, we know $p_{1 H}^{\max }=E V$ and $p_{1 L}^{\max }=L+q(H-L)\left[(2 N-T) /\left(2 N-S_{1}\right)\right]$, where $S_{1}$ denotes the seller's limit on advance sales at $p_{1}^{\max }$. This limit is constrainted to be feasible, i.e., $0 \leq S_{1} \leq \min [T, N]$. Note, given these conditions, $S_{1}$ is the actual sales in Period 1 because the seller adopts a price $p_{1}^{\max }$. We now find the optimal $S_{1}$ for each spot strategy for different capacity conditions. Then, we find the optimal global strategy. Let $L>c$.

A High Spot Price Strategy: $\left\{p_{2}=H\right\}$. The profits from this strategy are $\pi_{H}=\left(p_{1 H}^{\max }-c\right) S_{1}+(H-c) \min \left[q\left(2 N-S_{1}\right),\left(T-S_{1}\right)\right]$ Sales in Period 1 are $S_{1}$ and sales in Period 2 are the smaller of the number of buyers who enter Period 2 with high valuations, $q(2 N-$ $\left.S_{1}\right)$, and the remaining capacity, $\left(T-S_{1}\right)$. There are two cases: (a) $T-S_{1} \leq q\left(2 N-S_{1}\right)$ or $S_{1} \geq \hat{S}$ and (b) $T-S_{1} \geq q\left(2 N-S_{1}\right)$ or $S_{1}$ $\leq \hat{S}$, where $\hat{S}=(T-2 q N) /(1-q)$. Let $S_{1}^{*}$ be the optimal level of $S_{1}$, then the profits for these two cases are:

\begin{tabular}{|c|c|c|}
\hline Case & Total Profits $\left(p_{2}=H\right)$ & $\begin{array}{l}\text { Optimal Value of } \\
S_{1}\end{array}$ \\
\hline$S_{1} \geq \hat{S}$ & $\begin{aligned} & \pi_{H}=(H-c)\left(T-S_{1}\right) \\
&+\left(p_{1 H}^{\max }-c\right) S_{1} \\
&\left(\text { Note }\left(p_{1 H}^{\max }-c\right)<(H-c)\right)\end{aligned}$ & $\begin{aligned} S_{1}^{*}= & \min \left[S_{1}\right] \text { because } \pi_{H} \\
& \text { decreases with } S_{1}\end{aligned}$ \\
\hline$S_{1} \leq \hat{S}$ & $\begin{aligned} & \pi_{H}=(H-c) q\left(2 N-S_{1}\right) \\
&+\left(p_{1 H}^{\max }-c\right) S_{1} \\
&\left(\text { Note }\left(p_{1 H}^{\max }-c\right)>q(H-c)\right)\end{aligned}$ & $\begin{aligned} S_{1}^{*}= & \max \left[S_{1}\right] \text { because } \pi_{H} \\
& \text { increases with } S_{1}\end{aligned}$ \\
\hline
\end{tabular}

There are three possible ranges for $\hat{S}$. Rewriting the preceding table for these ranges we get:

\begin{tabular}{llc}
\hline \multicolumn{1}{c}{ Range } & Optimal $S_{1}$ & Total Profits $\left(p_{2}=H\right)$ \\
\hline $\begin{array}{l}0 \leq \hat{S} \\
\leq \min \{T, N\}\end{array}$ & $S_{1}^{*}=\hat{S}$ & $\pi_{H}=(H-c)(T-\hat{S})+\left(p_{1 H}^{\max }-c\right) \hat{S}$ \\
$\hat{S} \leq 0$ & $S_{1}^{*}=0$ & $\begin{array}{r}\pi_{H}=(H-c) T \\
\text { Note that if } \hat{S} \leq 0, \text { then } T \leq 2 q N .\end{array}$ \\
$\hat{S} \geq \min \{T, N\}$ & $S_{1}^{*}=\min \{T, N\} \quad \pi_{H}=\left\{\begin{array}{c}\left(p_{1 H}^{\max }-c\right) \min \left\{T, q\left(2 N-S_{1}^{*}\right)\right\} \\
\text { if } T \leq N \\
(H-c) \min \{T-N, q N\} \\
+\left(p_{1 H}^{\max }-c\right) N \\
\text { if } N \leq T\end{array}\right.$ \\
\hline
\end{tabular}

Note that when $\hat{S} \geq \min \{T, N\}$, it is not possible for $T \leq N$ because

$$
T \leq N \rightarrow \hat{S}=\frac{T-2 q N}{1-q} \leq\left(\frac{1-2 q}{1-q}\right) T \leq T .
$$

Hence, $T \geq N$ is the only case when $\hat{S} \geq \min \{T, N\}$. The table simplifies:

\begin{tabular}{llc}
\hline \multicolumn{1}{c}{ Range } & \multicolumn{1}{c}{ Optimal $S_{1}$} & Total Profits $\left(p_{2}=H\right)$ \\
\hline $0 \leq \hat{S} \leq N$ & $S_{1}^{*}=\hat{S}$ & $\pi_{H}=(H-c)(T-\hat{S})+\left(p_{1 H}^{\max }-c\right) \hat{S}$ \\
$\hat{S} \leq 0$ & $S_{1}^{*}=0$ & $\pi_{H}=(H-c) T$ \\
$\hat{S} \geq N$ & $S_{1}^{*}=\min \{T, N\}$ & $\pi_{H}=\begin{array}{l}(H-c) \min \{T-N, q N\} \\
\end{array}$ \\
& & $+\left(p_{1 H}^{\max }-c\right) N$ \\
\hline
\end{tabular}

We can rewrite the conditions in terms of capacity: $\hat{S} \leq 0$ implies $T \leq 2 q N ; 0 \leq \hat{S} \leq N$ implies $2 q N \leq T \leq(1+q) N ;$ and $\hat{S} \geq N$ implies $T \geq(1+q) N$. Rewriting the table in terms of capacity and finding the maximum profits, $\pi_{H}^{*}$ :

\begin{tabular}{|c|c|c|}
\hline Condition & $\begin{array}{l}\text { Optimal } \\
S_{1}=S_{1}^{*}\end{array}$ & Maximum Profit $\left(p_{2}=H\right)$ \\
\hline $\begin{array}{l}\text { Very small capacity } \\
\qquad(T \leq 2 q N)\end{array}$ & $S_{1}^{*}=0$ & $\pi_{H}^{*}=(H-c) T$ \\
\hline $\begin{array}{l}\text { Small capacity } \\
\qquad(2 q N<T \leq(1+q) N)\end{array}$ & $S_{1}^{*}=\hat{S}$ & $\begin{aligned} \pi_{H}^{*}= & (H-c)(T-\hat{S}) \\
& +\left(p_{1 H}^{\max }-c\right) \hat{S}\end{aligned}$ \\
\hline $\begin{array}{l}\text { Medium capacity } \\
\qquad \begin{array}{l}((1+q) N<T \\
\left.\quad \leq\left[1+\left(\frac{H-c}{L-c}\right) q\right] N\right)\end{array}\end{array}$ & $S_{1}^{*}=N$ & $\begin{aligned} & \pi_{H}^{*}=(H-c) q N \\
&+\left(p_{1 H}^{\max }-c\right) N \\
& \text { because } T \geq(1+q) N \\
& \rightarrow q N=\min \{T-N, q N\}\end{aligned}$ \\
\hline
\end{tabular}

Large capacity

$$
\begin{gathered}
\left(\left[1+\left(\frac{H-c}{L-c}\right) q\right] N\right. \\
<T<2 N)
\end{gathered}
$$

This case exists only if

$$
\left(\frac{H-c}{L-c}\right) q<1
$$

Finally, recall that high spot strategies must be credible. Credibility requires $(H-c) \min \left[q\left(2 N-S_{1}\right),\left(T-S_{1}\right)\right] \geq(L-c)\left(T-S_{1}\right)$. When capacity is very small, $S_{1}^{*}=0$, then the credibility condition is $(H-c) \min [2 N q, T] \geq(L-c) T$. Given $T \leq 2 q N$, this condition reduces to $(H-c) T \geq(L-c) T$, which is always true. When capacity is small, $S_{1}^{*}=\hat{S}$, then the credibility condition is $(H-c) \min [q(2 N$ $-\hat{S}), T-\hat{S}] \geq(L-c)(T-\hat{S})$. The definition of $\hat{S}$ implies $q(2 N-$ $\hat{S})=T-\hat{S}$, so the credibility condition reduces to $(H-c)(T-\hat{S})$ $>(L-c)(T-\hat{S})$, which is always true. When capacity is medium or large, $S_{1}^{*}=N$ and $q N<T-N$, so the credibility condition becomes $(H-c) q N \geq(L-c)(T-N)$. When capacity is medium, 


\section{XIE AND SHUGAN}

When and How to Advance Sell

$T \leq\{1+[(H-c) /(L-c)] q\} N$. Rearranging, we find $(H-c) q N \geq$ $(L-c)(T-N)$, so the credibility condition holds. When capacity is large $(T>\{1+[(H-c) /(L-c)] q\} N)$ we find $(H-c) q N<(L$ $-c)(T-N)$. So, the credibility condition fails. Note that credibility cannot be established in the large-capacity case by selling more in Period 1 because advance sales are limited to $N$.

Low Spot Price Strategy: $\left\{p_{2}=L\right\}$. The profit of this strategy is $\pi_{L}=\left(p_{1 L}^{\max }-c\right) S_{1}+(L-c) \min \left[2 N-S_{1}, T-S_{1}\right]$. Given $T<2 N$, we get $\pi_{L}=\left(p_{1 L}^{\max }-c\right) S_{1}+(L-c)\left(T-S_{1}\right)$. Theorem 6 shows that $p_{1 L}^{\max }=L+q(H-L)\left[(2 N-T) /\left(2 N-S_{1}\right)\right]$. Substituting,

$$
\pi_{L}=\left[L+q(H-L)\left(\frac{2 N-T}{2 N-S_{1}}\right)-c\right] S_{1}+(L-c)\left(T-S_{1}\right) .
$$

Simplifying,

$$
\pi_{L}=(H-L) q(2 N-T) \frac{S_{1}}{2 N-S_{1}}+(L-c) T .
$$

Given $\pi_{L}\left(S_{1}\right)$ is increasing with $S_{1}$, then $S_{1}^{*}=\min \{T, N\}$. We have two cases $S_{1}^{*}=T$ and $S_{1}^{*}=N$. However, when capacity is medium or large, then $T>(1+q) N \rightarrow T \geq N \rightarrow S_{1}^{*}=N$. Hence, the maximum profits for different levels of capacity are:

\begin{tabular}{ccc}
\hline Condition & $\begin{array}{l}\text { Optimal } \\
S_{1}=S_{1}^{*}\end{array}$ & Maximum Profit $\left(p_{2}=L\right)$ \\
\hline Very small and small capacity $(T \leq(1+q) N)$ & $S_{1}^{*}= \begin{cases}T & \text { if } T \leq N \\
N & \text { if } T \geq N\end{cases}$ & $\pi_{L}=\left\{\begin{array}{l}\left(p_{1 L}^{\max }-c\right) T \\
(L-c)(T-N)+\left(p_{1 L}^{\max }-c\right) N \quad \text { if } T \geq N\end{array}\right.$ \\
Medium and large capacity $(T>(1+q) N)$ & $S_{1}^{*}=N$ & $\pi_{L}=(L-c)(T-N)+\left(p_{L}^{\max }-C\right) N$ \\
\hline
\end{tabular}

Comparison. Now we compare the profits of the two strategies, $p_{2}=H$ and $p_{2}=L$, at each level of capacity:

\section{Condition}

Very small capacity $(T \leq 2 q N)$

Small capacity $(2 q N<T \leq(1+q) N)$

Medium capacity

$$
\left((1+q) N<T \leq\left[1+\left(\frac{H-c}{L-c}\right) q\right] N\right)
$$

Comparisons $\left(\Delta=\pi_{H}-\pi_{L}\right)$

$$
\begin{aligned}
& \Delta= \begin{cases}(H-c) T-\left(p_{1 L}^{\max }-c\right) T & \text { if } T \leq N \\
(H-c) T-(L-c)(T-N)-\left(p_{1 L}^{\max }-c\right) N & \text { if } T \geq N\end{cases} \\
& \Delta= \begin{cases}(H-c)(T-\hat{S})+\left(p_{1 H}^{\max }-c\right) \hat{S}-\left(p_{1 L}^{\max }-c\right) T & \text { if } T \leq N \\
(H-c)(T-\hat{S})+\left(p_{1 H}^{\max }-c\right) \hat{S}-(L-c)(T-N)-\left(p_{1 L}^{\max }-c\right) N & \text { if } T \geq N\end{cases} \\
& \Delta=(H-c) q N+\left(p_{1 H}^{\max }-c\right) N-(L-c)(T-N)-\left(p_{1 L}^{\max }-c\right) N
\end{aligned}
$$

Note that a high spot price is not applicable for the case of large capacity.

Simplifying (noting that $L \leq p_{1 L}^{\max } \leq p_{1 H}^{\max }<H, 0 \leq \hat{S} \leq N, 0 \leq \hat{S} \leq T$ ),

\section{Condition}

Very small capacity $(T \leq 2 q N)$

Small capacity $(2 q N<T \leq(1+q) N)$

Medium capacity

$$
\left((1+q) N<T \leq\left[1+\left(\frac{H-c}{L-c}\right) q\right] N\right)
$$

Comparisons $\left(\Delta=\pi_{H}-\pi_{L}\right)$

$$
\begin{aligned}
\Delta & = \begin{cases}\left(H-p_{L}^{\max }\right) T>0 & \text { if } T \leq N \\
(H-L) T-\left(p_{L}^{\max }-L\right) N>0 & \text { if } T \geq N\end{cases} \\
\Delta & = \begin{cases}\left(H-p_{L}^{\max }\right) T-\left(H-p_{1 H}^{\max }\right) \hat{S}>0 & \text { if } T \leq N \\
(H-L) T-\left(p_{L}^{\max }-L\right) N-\left(H-p_{1 H}^{\max }\right) \hat{S}>0 & \text { if } T \geq N\end{cases} \\
\Delta & =q(H-L) T-L[T-(1+q) N]+c[T-(1+q) N] \\
& =[q(H-L)-(L-c)] T+(L-c)(1+q) N \\
& >[q(H-L)-(L-c)](1+q) N+(L-c)(1+q) N=q(H-L)(1+q) N>0
\end{aligned}
$$

Remember, with a large capacity, $\{1+[(H-c)(L-c)] q\} N \leq T<2 N$, a high spot price strategy is not credible. Hence, a low spot price strategy is optimal to the case of large capacity. 
Given $p_{1 H}^{\max }=E V$, and $p_{1 L}^{\max }=\hat{p}_{1}=L+q(H-L)[1-(T-N) / N]$, we have proved:

\begin{tabular}{|c|c|}
\hline Condition & Optimal Strategy \\
\hline Very small capacity $(T \leq 2 q N)$ & A high spot price strategy is optimal $\rightarrow$ No advance sales: $\left\{p_{2}^{*}=H, S_{1}^{*}=0\right\}$ \\
\hline Small capacity $(2 q N<T \leq(1+q) N)$ & $\begin{array}{l}\text { A high spot price strategy is optimal } \rightarrow \text { Discounted advance selling with restricted advance } \\
\text { sales: } \\
\left\{p_{1}^{*}=E V, p_{2}^{*}=H, S_{1}^{*}=\frac{T-2 q N}{1-q}\right\}\end{array}$ \\
\hline $\begin{array}{l}\text { Medium capacity } \\
\qquad\left((1+q) N<T \leq\left[1+\left(\frac{H-c}{L-c}\right) q\right] N\right)\end{array}$ & $\begin{array}{l}\text { A high spot price strategy is optimal } \rightarrow \text { Discounted advance selling with restricted advance } \\
\text { sales: } \\
\left.p_{1}^{*}=E V, p_{2}^{*}=H, S_{1}^{*}=N\right\}\end{array}$ \\
\hline $\begin{array}{l}\text { Large capacity } \\
\left(\left[1+\left(\frac{H-c}{L-c}\right) q\right] N \leq T<2 N\right)\end{array}$ & $\begin{array}{l}\text { A low spot price strategy is optimal } \rightarrow \text { Premium advance selling without restricted advance } \\
\text { sales: } \\
\left\{p_{1}^{*}=\hat{p}_{1}, p_{2}^{*}=L, S_{1}^{*}=N\right\}\end{array}$ \\
\hline
\end{tabular}

Theorem 7 follows.

Proof of Table 4 (Impact of Risk Aversion). Recall Equation (3) in the paper

$$
\begin{gathered}
q U_{F}\left(w-p_{1}^{\max }\right)+(1-q) U_{U}\left(w-p_{1}^{\max }\right) \\
=q U_{F}(w-H)+(1-q) U_{U}(w-L) .
\end{gathered}
$$

Case 1:

$$
U_{j}(x)= \begin{cases}k+x & \text { if } j=F \\ x & \text { otherwise. }\end{cases}
$$

Equation (3) becomes:

$$
\begin{aligned}
& q\left(k+w-p_{1}^{\max }\right)+(1-q)\left(w-p_{1}^{\max }\right) \\
& \quad=q(k+w-H)+(1-q)(w-L) .
\end{aligned}
$$

Solving for $p_{1}^{\max }: p_{1}^{\max }=q H+(1-q) L$.

Case 2.

$$
U_{j}(x)= \begin{cases}k+\sqrt{x} & \text { if } j=F \\ \sqrt{x} & \text { otherwise. }\end{cases}
$$

Equation (3) becomes:

$$
\begin{aligned}
q( & \left.+\sqrt{w-p_{1}^{\max }}\right)+(1-q) \sqrt{w-p_{1}^{\max }} \\
& =q(k+\sqrt{w-H})+(1-q) \sqrt{w-L} .
\end{aligned}
$$

Solving for $p_{1}^{\max }: p_{1}^{\max }=w-[q \sqrt{w-H}+(1-q) \sqrt{w-L}]^{2}$. Case 3.

$$
U_{j}(x)= \begin{cases}k+\log (x) & \text { if } j=F \\ \log (x) & \text { otherwise. }\end{cases}
$$

Equation (3) becomes:

$$
\begin{aligned}
& q\left[k+\log \left(w-p_{1}^{\max }\right)\right]+(1-q) \log \left[w-k+\log \left(w-p_{1}^{\max }\right)\right] \\
& =q[k+\log (w-H)]+(1-q) \log (w-L) .
\end{aligned}
$$

Solving for $p_{1}^{\max }: p_{1}^{\max }=w-e^{q \log (w-H)+(1-q) \log (w-L)}$.

Case 4.

$$
U_{j}(x)= \begin{cases}\sqrt{k+x} & \text { if } j=F \\ \sqrt{x} & \text { otherwise. }\end{cases}
$$

Equation (3) becomes:

$$
\begin{aligned}
& q\left(\sqrt{k+w-p_{1}^{\max }}\right)+(1-q) \sqrt{w-p_{1}^{\max }} \\
& \quad=q(\sqrt{k+w-H})+(1-q) \sqrt{w-L} .
\end{aligned}
$$

Table 4 follows.

Proof of Theorem 8 (Optimal Pricing with Exogenous Credibility). Spot Selling Only. As shown in Theorem 2, the optimal prices and profits are

$$
p_{2}^{*}= \begin{cases}H & \text { if } c \geq \underline{c} \\ L & \text { otherwise, }\end{cases}
$$

where $\underline{c}=(L-q H) /(1-q$,$) and$

$$
\pi_{S}= \begin{cases}q(H-c) 2 N & \text { if } p_{2}^{*}=H \\ (L-c) 2 N & \text { if } p_{2}^{*}=L .\end{cases}
$$

Advance Selling. With exogenous credibility, sellers have two possible optimal advance selling strategies: (a) a high spot-price strategy $\left\{p_{1}=E V, p_{2}=H\right\}$, and (b) a low spot-price strategy $\left\{p_{1}=L\right.$, $\left.p_{2}=L\right\}$. Let $\pi_{H}$ and $\pi_{L}$ be the profits of the high spot-price advance selling and the low spot-price advance selling strategies, respectively. Let $\pi_{S}$ be the profit of spot-only strategy.

High Spot-price Advance Selling. $\pi_{H}=[E V-c] N+q(H-c) N$. 
Let $\underset{c}{c}=[(1+q) L-2 q H] /(1-q)$. Note $\underline{c}-\underline{\underline{c}}=[q(H-L)] /(1$ $-q)>0$

$\pi_{A}-\pi_{S}$

$$
= \begin{cases}(L-c)(1-q) & \text { if } p_{2}^{*}=H(\text { or } L>c>\underline{c}) \\ 2 q H-(1+q) L+(1-q) c & \text { if } p_{2}^{*}=L(\text { or } \underline{c}>c>\underline{\underline{c}}) .\end{cases}
$$

Thus,

$$
\pi_{A}-\pi_{S} \begin{cases}>0 & \text { if } L>c>\underline{\underline{c}} \\ \leq 0 & \text { otherwise. }\end{cases}
$$

Low Spot-price Advance Selling. $\pi_{A}=2(L-c) N$, so

$$
\pi_{A}-\pi_{S} \begin{cases}<0 & \text { if } p_{2}^{*}=H \\ =0 & \text { if } p_{2}^{*}=L\end{cases}
$$

Theorem 8 follows.

Proof of Thorem 9 (Refunds). Advance Selling without Refunds. $p_{1}^{\max }\left(\right.$ No Refund) $=\left\{\begin{array}{ll}q_{H} H+q_{L} L & \text { if } p_{2}^{*}=H \\ \left(q_{H}+q_{L}\right) L & \text { if } p_{2}^{*}=L\end{array} \quad\right.$ (See Equation 5).

Thus,

$\pi_{A}($ No Refund $)= \begin{cases}{\left[q_{H} H+q_{L} L-c\right] N+q_{H}(H-c) N} & \text { if } p_{2}^{*}=H \\ 2\left(q_{H}+q_{L}\right)(L-c) N & \text { if } p_{2}^{*}=L .\end{cases}$

Advance Selling with Refunds.

$p_{1}^{\max }($ Refund $)=\left\{\begin{array}{ll}q_{H} H+q_{L} L+q_{0} R & \text { if } p_{2}^{*}=H \\ \left(q_{H}+q_{L}\right) L+q_{0} R & \text { if } p_{2}^{*}=L\end{array} \quad\right.$ (See Equation 6).

Thus,

$$
\begin{aligned}
& \pi_{A}(\text { Refund }) \\
& \quad= \begin{cases}{\left[q_{H}(H-c)+q_{L}(L-c)\right] N+\left[q_{H}(H-c)\right] N} & \text { if } p_{2}^{*}=H \\
2\left(q_{H}+q_{L}\right)(L-c) N & \text { if } p_{2}^{*}=L .\end{cases}
\end{aligned}
$$

Spot Selling Only.

$$
\pi_{S}= \begin{cases}2 q_{H}(H-c) N & \text { if } p_{2}^{*}=H \\ 2\left(q_{H}+q_{L}\right)(L-c) N & \text { if } p_{2}^{*}=L .\end{cases}
$$

Comparison.

$$
\begin{array}{r}
\pi_{A} \text { (Refund) }-\pi_{S}= \begin{cases}q_{L}(L-c) N & \text { if } p_{2}^{*}=H \\
0 & \text { if } p_{2}^{*}=L,\end{cases} \\
\left.\pi_{A} \text { (Refund }\right)-\pi_{A}(\text { No Refund })= \begin{cases}q_{0} c N & \text { if } p_{2}^{*}=H \\
0 & \text { if } p_{2}^{*}=L .\end{cases}
\end{array}
$$

Theorem 9 follows.

\section{References}

Ansari, Asim, S. Siddarth, Charles B. Weinberg. 1996. Pricing a bundle of products or services: The case of nonprofits. J. Marketing Res. 33 (February) 86-93.
Bettman, James R., Mary Frances Luce, John W. Payne. 1998. Constructive consumer choice processes. J. Consumer Res. 25(3) 187217.

Biyalogorski, E., Z. Carmon, G. Fruchter, E. Gerstner. 1999. Overselling with opportunistic cancellations. Marketing Sci. 18(4) 605-610.

Blattberg, Robert C., Gary Eppen, Joshua Lieberman. 1981. A theoretical and empirical evaluation of price deals for consumer non-durables. J. Marketing 45(1) 116-129.

Bockenholt, Ulf, William R. Dillon. 1997. Some new methods for an old problem: Modeling preference changes and competitive market structures in pretest market data. J. Marketing Res. XXXIV 130-142.

Coase, Ronald H. 1972. Durability and monopoly. J. Law and Econom. 15 (April) 143-149.

Cook, Phillip J., Daniel A. Graham. 1977. The demand for insurance and protection: The case of irreplaceable commodities. Quart. J. Econom. 91 (Feb.) 143-154.

Dana, James D. 1999. Using yield management to shift demand when the peak time is unknown. RAND J. Econom. 30(3) 456474 .

Dawe, Tony. 2000. Lap up a little luxury. The London Times (October 26).

Desiraju, Ramarao, Steven Shugan. 1999. Strategic service pricing and yield management. J. Marketing 63 (Winter) 44-56.

Eliashberg, Jehoshua, Jagmohan S. Raju, Wilfred Amaldoss. 1998. Estimating the demand for sell-through home videos: A model and an empirical application. Working paper 98-024, Wharton Marketing Department, University of Pennsylvania, Philadelphia, PA.

Fishburn, Peter C. 1974. On the foundations of decision making under uncertainty. M. Balch, D. McFadden, S. Wu, eds. Essays on Economic Behavior Under Uncertainty. North Holland, Amsterdam, The Netherlands.

Fruchter, Gila, Eitan Gerstner. 1999. Selling with satisfaction guaranteed. J. Service Res. 1(4) 313-323.

Gale, Ian, Thomas Holmes. 1993. Advanced purchase discounts and monopoly allocation of capacity. Amer. Econom. Rev. 83 135-146.

Gerstner, Eitan, James D. Hess. 1987. Why do hot dogs come in packs of $10 \mathrm{~s}$ and buns $8 \mathrm{~s}$ or $12 \mathrm{~s}$ ? A demand side explanation. J. Bus. 60(4) 491-517.

Greenleaf, Eric A., Donald R. Lehmann. 1995. Reasons for substantial delay in consumer decision making. J. Consumer Res. 22(2) 186199.

Hauser, John R., Birger Wernerfelt. 1990. An evaluation cost model of consideration sets. J. Consumer Res. 16(March) 393-408.

Hayes, Beth. 1987. Competition and two-part tariffs. J. Bus. 60(1) $41-54$.

Jeuland, Abel P., Chakravarthi Narasimhan. 1985. Dealing-temporary price cuts-by seller as a buyer discrimination mechanism. J. Bus. 58(3) 295-308.

Johnson, Michael D., Eugene W. Anderson, Claes Fornell. 1995. Rational and adaptive performance expectations in a customer satisfaction framework. J. Consumer Res. 21(4) 695-707. 
Kahn, Barbara E., Donald R. Lehmann. 1991. Modeling choice among assortments. J. Retailing 67(Fall) 274-299.

Krider, Robert E. and Charles B. Weinberg. 1998. Competitive dynamics and the introduction of new products: The motion picture timing game. J. Marketing Res. 35(1) 1-15.

Kreps, David M. 1979. A representation theorem for preferemce for flexibility. Econometrica 47(3) 565-577.

Lal, Rajiv, V. Padmanabhan. 1995. Competitive response and equilibria. Marketing Sci. 14(3, Part 2) 101-108.

Lazear, Edward P. 1986. Retail pricing and clearance sales. Amer. Econom. Rev. 76 14-32.

Lollar, Coleman. 1992. Trade secrets of the travel game. Working Woman 17(May) 94.

Malinvaud, Edmond 1972. Lectures on Micro-Economic Theory. NorthHolland Publishing Company, Amsterdam, The Netherlands.

McAlister, Leigh, Edgar Pessemier. 1982. Variety seeking behavior: An interdisciplinary review. J. Consumer Res. 9(3) 311-322.

McVea, Robert. 1997. Wild west lives again in suburbs. Chicago Tribune (August 3).

Melendez, Michele M. 1997. Underground dance scene turn fans into ravin'. The Plain Dealer, Cleveland, $\mathrm{OH}$.

Mitchell, Deborah J., Barbara E. Kahn, Susan C. Knasko. 1995. There's something in the air: Effects of ambient odor on consumer decision making. J. Consumer Res. 22(September) 229-238.

Padmanabhan, V., Ivan P.L. Png. 1995. Returns policies: Make money by making good. Sloan Management Rev. 37(1) 65-72.

— Surendra Rajiv, Kannan Srinivasan. 1997. New products, upgrades, and new releases: A rationale for sequential product introduction. J. Marketing Res. XXXIV(Fall) 456-472.

Plummer, M. L., R. C. Hartman. 1986. Option value: A general approach. Econom. Inquiry (October) 24 455-472.

Png, Ivan P.L. 1989. Reservations: Customer insurance in the marketing of capacity. Marketing Sci. 8(3) 248-264.

Radas, Sonja, Steven M. Shugan. 1998a. Seasonal marketing and timing new product introductions. J. Marketing Res. 35(August) 296-315.

1998b. Managing service demand: Shifting and bundling. J. Service Res. 1(1) 47-64.
Rust, Roland T., Richard L. Oliver. 1994. Service quality, insights and managerial implications from the frontier. Service Quality: New Directions in Theory and Practice. Sage Publications, Newbury Park, CA.

Savage, Leonard J. 1954. The Foundations of Statistics. Dover, New York.

Shugan, Steven M. 1994. Explanations for service growth. Richard Oliver, Roland Rust, eds. Service Quality. Sage Publications, Newbury Park, CA, 223-239.

— Jinhong Xie. 2000. Advance pricing of services and other implications of separating purchase and consumption. J. Service Res. 2(February) 227-239.

- -2001 . Advance-selling strategies with competition. Rev. Marketing Sci. working paper series: WP No. 2001213, http: / / roms.utdallas.edu/working_series.asp.

Simonson, Itamar, Russell S. Winer. 1992. The influence of purchase quantity and display format on consumer preference for variety. J. Consumer Res. 19(June) 133-138.

Sobel, Joel, Ichiro Takahashi. 1983. A multistage model of bargaining. Rev. Econom. Stud. 50(3) 411-426.

Srinivasan, V., William S. Lovejoy, David Beach. 1997. Integrated product design for marketability and manufacturing. J. Marketing Res. XXXIV (February) 154-163.

Stayman, Douglas M., Rohit Deshpande. 1989. Situational ethnicity and consumer behavior. J. Consumer Res. 16 (December) 361371.

Strandberg, Keith W. 1992. Systems now and future: Fare collection systems for mass transportation. Mass Transit 19(9-10) 47-51.

Wernerfelt, Birger. 1995. A rational reconstruction of the compromise effect: Using market data to infer utilities. J. Consumer Res. 4 (March) 627-633.

Working, Holbrook. 1953. Futures trading and hedging. Amer. Econom. Rev. 43 (June) 314-343.

Xie, Jinhong, Marvin A. Sirbu. 1995. Price competition and compatibility in the presence of network externalities. Management Sci. 41 (May) 909-926.

Zettelmeyer, Florian. 1996. The strategic use of consumer search cost. Ph.D. thesis, Sloan School of Management, Massachusetts Institute of Technology, Cambridge, MA.

This paper was received June 9, 2000, and was with the authors 5 months for 2 revisions; processed by James D. Hess. 